\title{
Regulatory T cell transfer ameliorates lymphedema and promotes lymphatic vessel function
}

\author{
Epameinondas Gousopoulos, ${ }^{1}$ Steven T. Proulx, ${ }^{1}$ Samia B. Bachmann, ${ }^{1}$ Jeannette Scholl, ${ }^{1}$ \\ Dimitris Dionyssiou, ${ }^{2}$ Efterpi Demiri, ${ }^{2}$ Cornelia Halin, ${ }^{1}$ Lothar C. Dieterich, ${ }^{1}$ and Michael Detmar ${ }^{1}$ \\ IInstitute of Pharmaceutical Sciences, ETH Zurich, Switzerland. ${ }^{2}$ Department of Plastic Surgery, Aristotle University of \\ Thessaloniki, Thessaloniki, Greece.
}

\begin{abstract}
Secondary lymphedema is a common postcancer treatment complication, but the underlying pathological processes are poorly understood and no curative treatment exists. To investigate lymphedema pathomechanisms, a top-down approach was applied, using genomic data and validating the role of a single target. RNA sequencing of lymphedematous mouse skin indicated upregulation of many $\mathrm{T}$ cell-related networks, and indeed depletion of $\mathrm{CD} 4^{+}$cells attenuated Iymphedema. The significant upregulation of Foxp3, a transcription factor specifically expressed by regulatory T cells (Tregs), along with other Treg-related genes, implied a potential role of Tregs in lymphedema. Indeed, increased infiltration of Tregs was identified in mouse lymphedematous skin and in human lymphedema specimens. To investigate the role of Tregs during disease progression, loss-of-function and gain-of-function studies were performed. Depletion of Tregs in transgenic mice with Tregs expressing the primate diphtheria toxin receptor and green fluorescent protein (Foxp3-DTR-GFP) mice led to exacerbated edema, concomitant with increased infiltration of immune cells and a mixed $\mathrm{T}_{\mathrm{H}} 1 / \mathrm{T}_{\mathrm{H}} 2$ cytokine profile. Conversely, expansion of Tregs using IL-2/antiIL-2 mAb complexes significantly reduced lymphedema development. Therapeutic application of adoptively transferred Tregs upon lymphedema establishment reversed all of the major hallmarks of lymphedema, including edema, inflammation, and fibrosis, and also promoted lymphatic drainage function. Collectively, our results reveal that Treg application constitutes a potential new curative treatment modality for lymphedema.
\end{abstract}

Conflict of interest: The authors have declared that no conflicts of interest exist.

Submitted: June 13, 2016 Accepted: September 6, 2016 Published: October 6, 2016

Reference information: JCI Insight. 2016;1(16):e89081. doi:10.1172/jci.insight.89081.

\section{Introduction}

Secondary lymphedema commonly arises as an iatrogenic complication following surgery and/or radiotherapy in cancer patients, due to the interruption or impairment of the lymphatic drainage system (1). Although breast cancer treatment with axillary lymph node dissection and/or radiotherapy is the leading cause, with an incidence rate up to $30 \%$ among breast cancer survivors (2), lymphedema may also appear after surgical removal of other solid tumors, such as gynecological tumors and melanomas, or even after seemingly minor interventions such as lymph node biopsies (3). Lymphedema patients experience progressive functional impairment of the diseased limb, characterized by swelling, leading to physical discomfort and disfigurement, sensory deficits, recurrent inflammation, fibrosis, impaired wound healing and, rarely, secondary tumors. Despite the large number of patients affected, little is known about the homeostatic perturbations underlying the development of this disease, and there are currently no curative therapies.

Although lymphatic vessel injury and dysfunction are considered to represent the primary defects underlying lymphedema development (4), the onset of lymphedema can be delayed by several years after surgery, and there is increasing evidence that inflammation strongly contributes to the pathogenesis (5-8). A recent microarray-based gene expression analysis of mouse lymphedematous tissue (7) indicated that lymphedema progression is characterized by intense inflammatory changes, and experimental studies by our group and others revealed a heterogeneous immune cell infiltrate in the lymphedematous tissue $(5,8)$. Indeed, depletion of specific immune cell populations using antibodies or genetic mouse models indicated that a reduction of macrophages (6) or of $\mathrm{CD}^{+}$cells $(6,8)$ ameliorates experimental lymphedema. Similarly, administration of COX-2 inhibitors can lessen the severity of the disease, although without full restoration of the functionality of the lymphatic network (5). 
The lymphatic system and the immune system are intimately connected, and recent studies have revealed that lymphatic vessels are dynamic structures that sensibly react to inflammatory stimuli. Whereas lymphatic drainage is increased during acute inflammation (9), probably to compensate for the increased fluid leakage from blood vessels, recent work by us and others has shown that chronic inflammation severely impairs lymphatic fluid drainage, promoting edema formation and accumulation of inflammatory mediators at the inflamed site $(9,10,11)$. Importantly, there is evidence that cytokines released by activated $\mathrm{T}_{\mathrm{H}} 1$ and $\mathrm{T}_{\mathrm{H}} 2$ cells negatively impact the lymphatic network, both at the morphological and functional level (12-14).

The aim of the present study was to investigate the pathomechanisms that lead to lymphedema, using a surgical mouse tail lymphedema model $(5,15)$ and RNA sequencing at 0,2 , and 6 weeks after lymphedema surgery. We found a significant upregulation of T cell-related networks at 2 and 6 weeks after surgery, with a particular upregulation of Foxp3 and other genes associated with regulatory $\mathrm{T}$ cells (Tregs), suggesting a potential role for Tregs in lymphedema pathogenesis. This was confirmed by immunostaining that revealed increased Treg numbers in experimental mouse lymphedema and also in skin samples of lymphedema patients. While global $\mathrm{CD}^{+}$cell depletion attenuated lymphedema development, in agreement with recent studies $(6,8)$, targeted depletion of Tregs exacerbated lymphedema and increased inflammation and $\mathrm{T}_{\mathrm{H}} 1$ and $\mathrm{T}_{\mathrm{H}} 2$ responses. Conversely, systemic Treg expansion using IL-2/anti-IL-2 mAb complexes reduced lymphedema development. Importantly, a therapeutic trial using adoptive Treg transfer at 1 week after lymphedema surgery revealed improvement of all of the major hallmarks of lymphedema, namely tissue swelling, inflammation, fibrosis, lymphatic vessel enlargement, and impaired lymphatic drainage function. Together, these results identify Treg application as a potential novel therapeutic modality for the treatment of lymphedema.

\section{Results}

Tissue inflammation in lymphedema is characterized by $T$ cell activation. To investigate potential pathomechanisms underlying lymphedema development, we employed a mouse tail lymphedema model and performed RNA sequencing on control (unoperated) and lymphedematous tail skin 2 and 6 weeks after surgery. Using a threshold of 0.5 ( $\log 2$ value) for expression level changes and a $P$ value of less than 0.05 , we found 381 genes uniquely upregulated and 229 gene downregulated 2 weeks after surgery, 990 genes uniquely upregulated and 744 downregulated at the 6-week time point, while 768 genes were commonly upregulated and 375 genes commonly downregulated at both time points (Figure 1, A and B, and Supplemental Data Files 1 and 2; supplemental material available online with this article; doi:10.1172/jci.insight.89081DS1). Evaluation of the key pathways modulated during lymphedema development using MetaCore indicated a significant upregulation of T cell-related networks (lymphocyte proliferation, T cell receptor [TCR] signaling; Figure 1C), suggesting increased T cell infiltration and activation during lymphedema development.

Depletion of $\mathrm{CD}^{+} T$ cells decreases lymphedema and improves lymphatic vessel function. Based on the results of the transcriptional profiling and the recently suggested role of $\mathrm{CD}^{+}$cells in lymphedema $(6,8)$, we first investigated the functional role of $\mathrm{CD}^{+} \mathrm{T}$ cells in this model. Depletion of $\mathrm{CD}^{+}$cells using CD4-specific antibodies resulted in significantly decreased tail volumes at 3 and 4 weeks after surgery under CD4 ${ }^{+}$ cell depletion $(P<0.01$ at both time points) (Figure 2, A and B). Since enlargement of lymphatic vessels represents a hallmark of lymphedema in this model (5), we next evaluated lymphatic vessel morphology by immunofluorescence staining of tail sections for the lymphatic marker LYVE-1. CD4 ${ }^{+}$cell depletion resulted in a significant reduction of the tissue area covered by lymphatic vessels (Figure 2, C and D). Concomitantly, expression of VEGF-C in the affected tissue was also reduced (Figure 2E). Since the presence of dilated lymphatic vessels has been associated with impaired lymphatic vessel transport function (16), a functional assessment of lymphatic vasculature transport was performed using noninvasive near-infrared (NIR) intravital microscopy. A PEGylated NIR dye that is selectively taken up by lymphatic vessels (17) was slowly perfused into the tip of the tails, and its transport to the edge of the surgical excision was monitored and quantified. Consistent with the morphological changes, depletion of $\mathrm{CD} 4^{+}$cells significantly improved lymphatic transport compared with the IgG-treated group (Figure 2F), as evidenced by an increased amount of dye reaching the honeycomb-shaped dermal lymphatic vessel network in close proximity to the site of surgery (Figure $2 \mathrm{G}$ ). Taken together, these data suggest that a $\mathrm{T}$ helper response contributes to lymphedema development and lymphatic dysfunction.

Increased Treg cell infiltrate in lymphedematous tissue. Foxp3, a gene expressed specifically by Tregs, was found in the RNA sequencing study to be among the most highly upregulated genes 2 and 6 weeks after surgery, representing the only transcription factor among those genes. Furthermore, there was an upreg- 
A

Up regulated genes

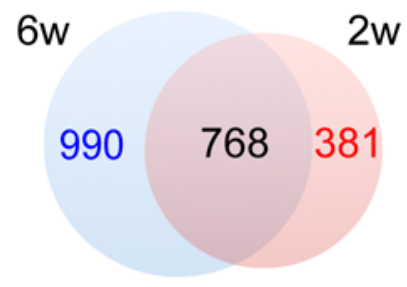

B Down regulated genes

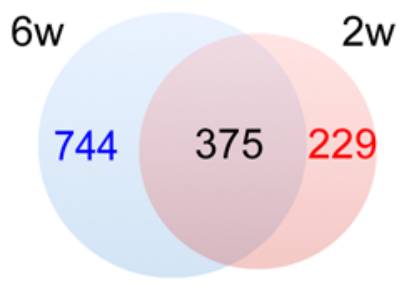

C

Gene Networks

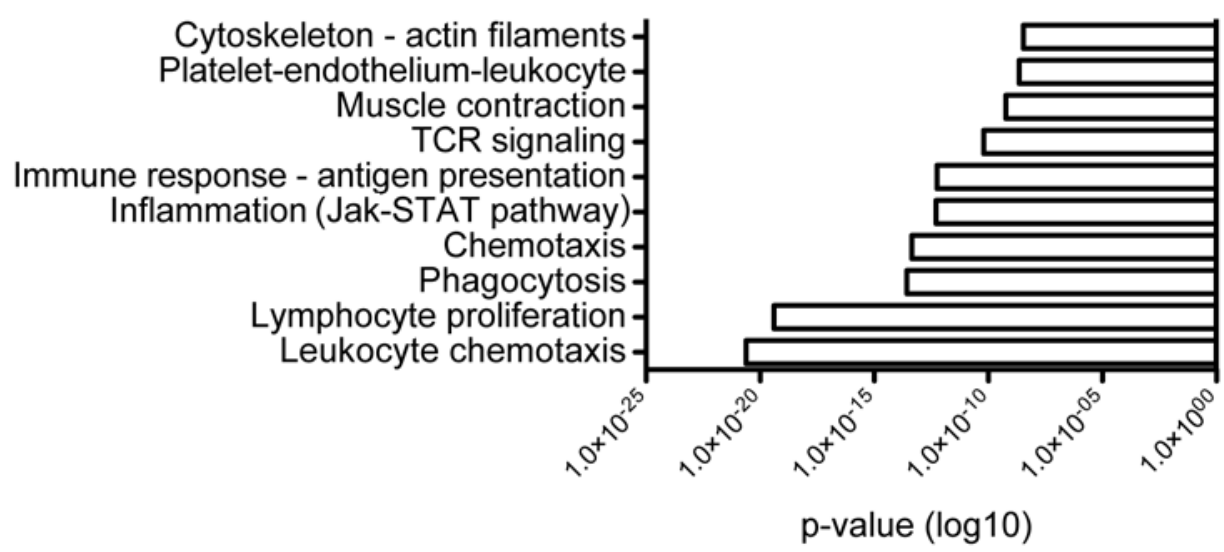

Figure 1. Lymphedema development is characterized by inflammation. (A) Schematic representation of the number of genes uniquely or commonly upregulated 2 and 6 weeks after surgery. (B) Schematic representation of the number of genes uniquely or commonly downregulated 2 and 6 weeks after surgery. (C) List of the 10 most significantly upregulated gene networks in lymphedema. Network analysis of the genes upregulated 2 and 6 weeks after surgery in comparison with control (unoperated) mice, according to RNA sequencing analysis, revealed a significant activation of inflammation- and T cell-related networks during lymphedema development ( $n=5$ per group per time point). RNA sequencing was performed once.

ulation of several other genes associated with activated Tregs, such as Icos, II2ra (CD25), and Lap (Figure $3 \mathrm{~A})$. Thus, we next investigated if this gene signature was reflected by an increased Treg infiltrate in the lymphedematous tissue. Immunofluorescence staining using transgenic mice with Tregs expressing the primate diphtheria toxin receptor and green fluorescent protein (Foxp3-DTR-GFP, referred to hereafter as DEREG mice) revealed a clear increase in Tregs both 2 and 6 weeks after surgery (Figure 3B). Flow cytometry indicated that the percentages of Tregs increased both among total single cells $(P<0.001)$ and within the $\mathrm{CD}^{+}$cell compartment $(P<0.05)$ at the 2-week time point (Figure $3, \mathrm{C}$ and $\mathrm{D}$ ). A systemic increase in circulating Tregs of about $40 \%(P<0.05)$ was noted 1 week after surgery in comparison with unoperated mice (Figure 3E), whereas circulating Treg numbers were not elevated 2 weeks after surgery, and no changes were observed in the amount of Tregs in the spleen at any time point. Notably, an increase in $\mathrm{CD}^{2} 5^{+}$cells (Figure 3, F and G) and $\mathrm{FOXP}^{+}$cells (Figure 3, H and I, and Supplemental Figure 1) was also detectable in skin biopsies of lymphedema patients $(P<0.001)$, highlighting the clinical relevance of these experimental data.

Depletion of Tregs aggravates inflammation and exacerbates lymphedema development. The increased infiltration of Tregs in the lymphedematous tissue led us to further investigate the role of this specific $\mathrm{T}$ cell subset in lymphedema progression. DEREG mice were used to deplete Tregs upon injection with diphtheria toxin. Using this model, we found that depletion of Tregs directly after surgical induction of lymphedema led to increased tail volumes 1 week after surgery (Figure 4A). Morphological evaluation of the lymphatic and blood vascular response did not reveal any obvious changes (Figure 4, B and C). Interestingly, expression levels of $I F N-\gamma, I L-13, I L-4$, and TGF- $\beta 1$, cytokines linked to reduced lymphatic vessel functionality and fibrosis development $(12,14,15,18)$, were significantly increased in tissue lysates 1 week after Treg depletion (Figure 4D), indicating a profound change in the tissue microenvironment to promote lymphatic 
A
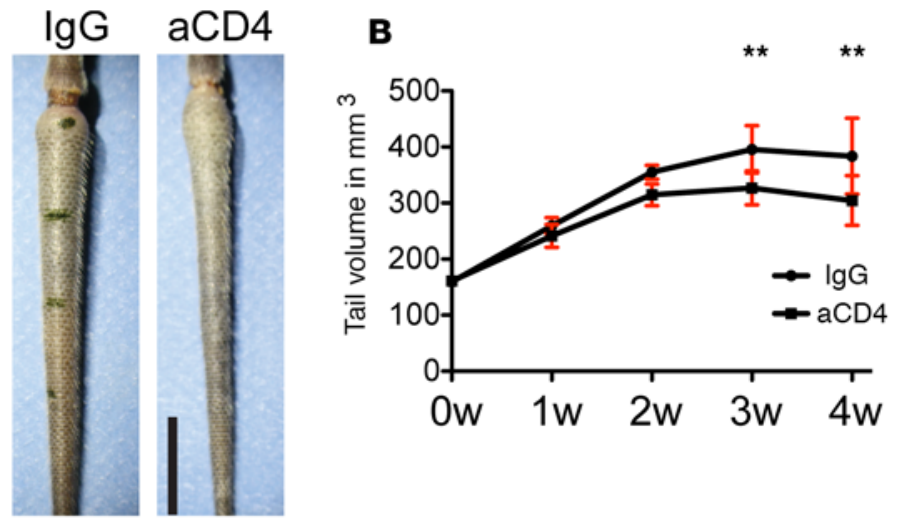

D

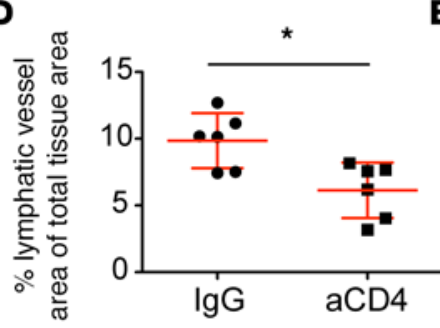

$\mathbf{E}$

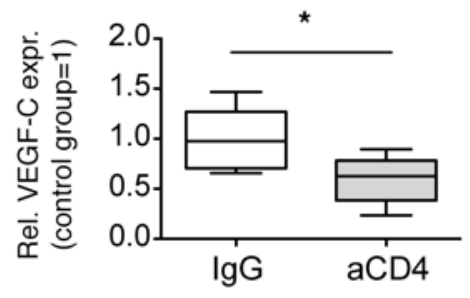

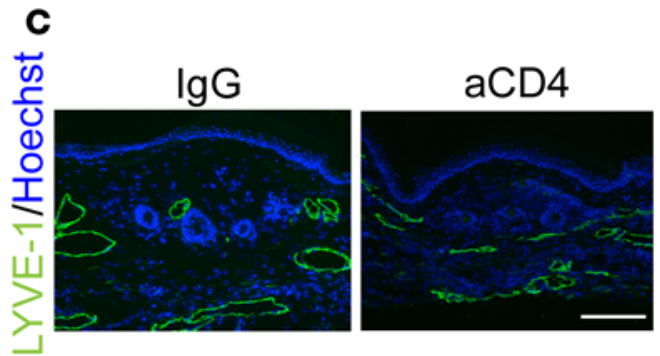
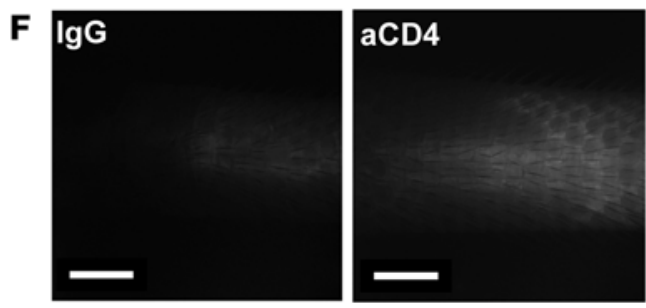

G

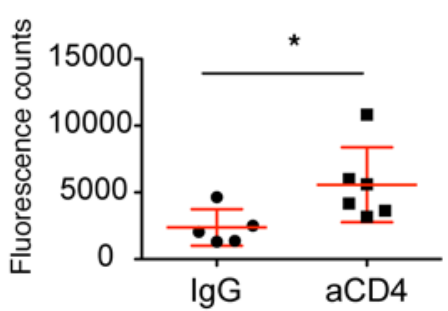

Figure 2. Depletion of CD4+ cells improves lymphedema and lymphatic function. (A) Representative photographs of tails of control and anti-CD4-treated (aCD4 in figure) mice. Scale bar: $1 \mathrm{~cm}$. (B) Depletion of CD4+ cells immediately following induction of lymphedema leads to significantly reduced tail volumes 3 and 4 weeks after surgery ( $n=5-6$ per group). (C) Representative micrographs depicting the significantly reduced lymphatic vascular dilation in the anti-CD4-treated group. Scale bar: $200 \mu \mathrm{m}$. (D) Quantification of tissue area covered by lymphatic vessels, including the luminal area. (E) qPCR analysis of VEGF-C mRNA expression in total tissue extracts. (F) Representative images of the tail lymphatic network close to the surgical site, visualized by uptake and transport of a lymphatic-specific fluorescent tracer 20 minutes after infusion near the tip of the tail. Scale bars: $2 \mathrm{~mm}$. (C) Quantification of lymphatic vascular transport based on fluorescence intensity revealed significantly improved lymphatic vessel function in the proximity of the surgical excision margin after anti-CD4 treatment ( $n=4-6$ per group, in at least 2 replicates). Statistical analysis was done with 2-way ANOVA with Bonferroni post-hoc test (B) or Student's $t$ test $(\mathbf{D}, \mathbf{E}$, and $\mathbf{G}),{ }^{*} P<0.05,{ }^{* *} P<0.01$.

dysfunction and fibrosis. There was a trend towards increased expression of $T N F-\alpha$ and $I L-10$, whereas $V E G F-C$ expression levels remained unchanged. Evaluation of infiltration of total leukocytes and macrophages in the lymphedematous tissue, using immunofluorescence staining, revealed that $\mathrm{CD}_{4} 5^{+}$and $\mathrm{CD}^{206}{ }^{+}$cells were significantly increased after Treg depletion $(P<0.05$ and $P<0.01$, respectively), and that $\mathrm{CD}_{68}{ }^{+}$macrophages showed a trend towards increased numbers (Figure 4, E and F), indicating an aggravated inflammatory response induced by the lack of Tregs.

Systemic expansion of Tregs by IL-2/anti-IL-2 mAb complexes reduces tail edema. Since absence of Tregs led to aggravated lymphedema, we next assessed the potential value of Treg amplification, using IL-2/ anti-IL-2 mAb complexes (IL2-c), which selectively increase the number of Tregs under basal conditions in mice (19). Mice were treated for 3 consecutive days with IL2-c prior to surgical lymphedema induction to avoid expansion of other activated $\mathrm{T}$ cells during the initial stages of the disease. At 1 week after surgery, the IL2-c-treated mice exhibited significantly reduced tail swelling $(P<0.05$; Figure $5 \mathrm{~A})$. Similar to the Treg depletion study, there were no significant morphological effects on the lymphatic or blood vasculature (Figure 5, B and C), whereas assessment of the inflammatory response by immunofluorescence staining showed a decrease in the $\mathrm{CD}_{4} 5^{+}, \mathrm{CD} 206^{+}$, and $\mathrm{CD} 68^{+}$infiltrates $(P<0.05)$ (Figure 5, D and E).

Adoptive Treg transfer reverses pathological manifestations of lymphedema. Treg amplification using IL2-c treatment before lymphedema surgery revealed a beneficial role of Tregs in the prevention of lymphedema. To investigate the efficacy of an increase in Tregs in a therapeutic setting with potential clinical application, we next performed adoptive transfer of Tregs in mice with established lymphedema. Tregs were expanded in vivo using IL2-c in donor mice and were isolated by FACS from the spleen and adoptively transferred into mice 1 
A

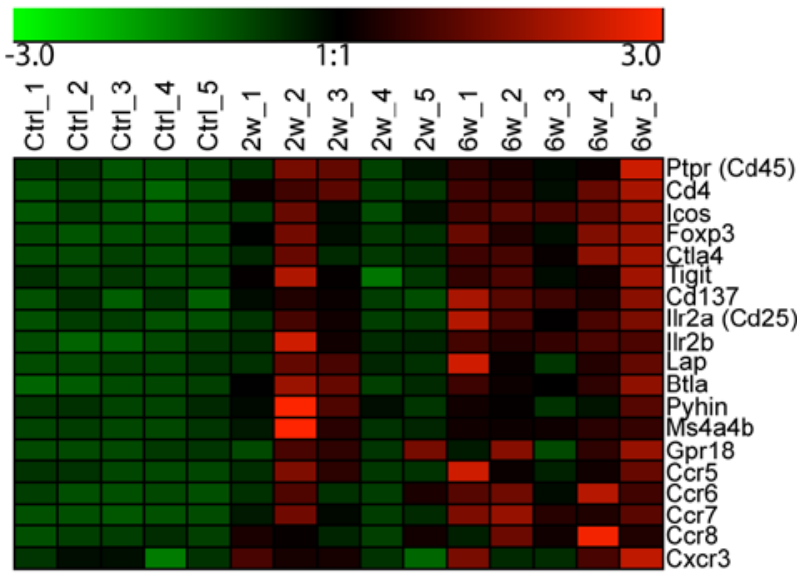

B
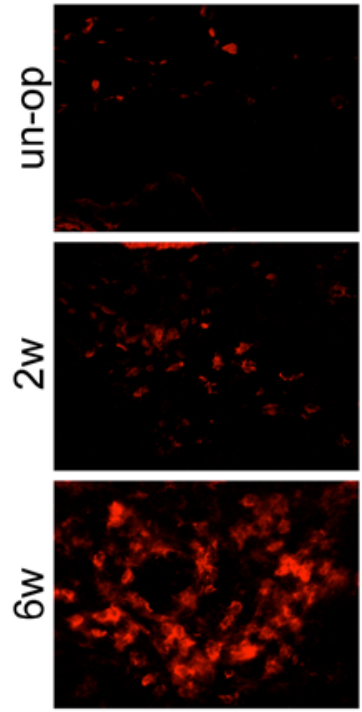

G

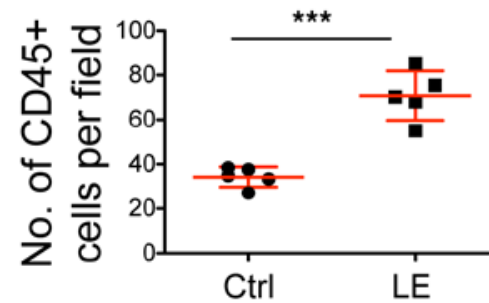

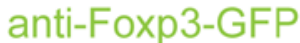
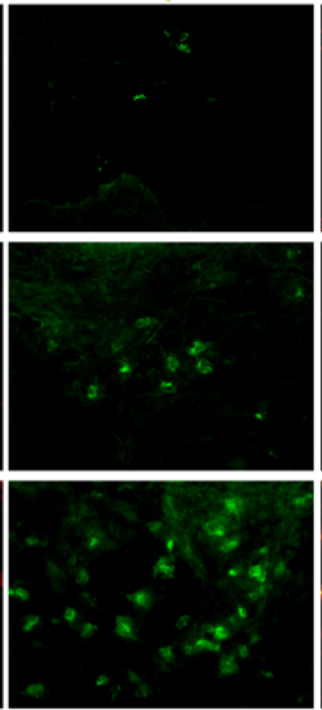

I

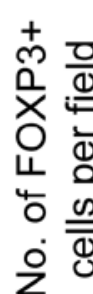

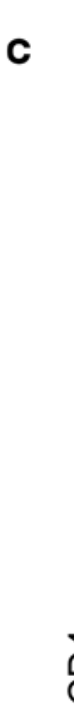

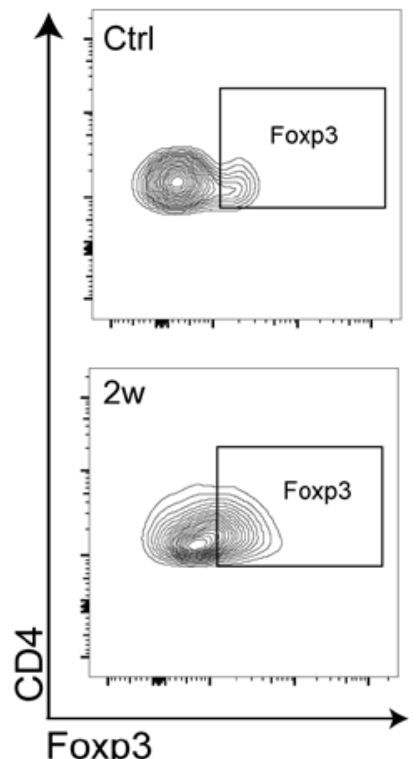

Foxp3
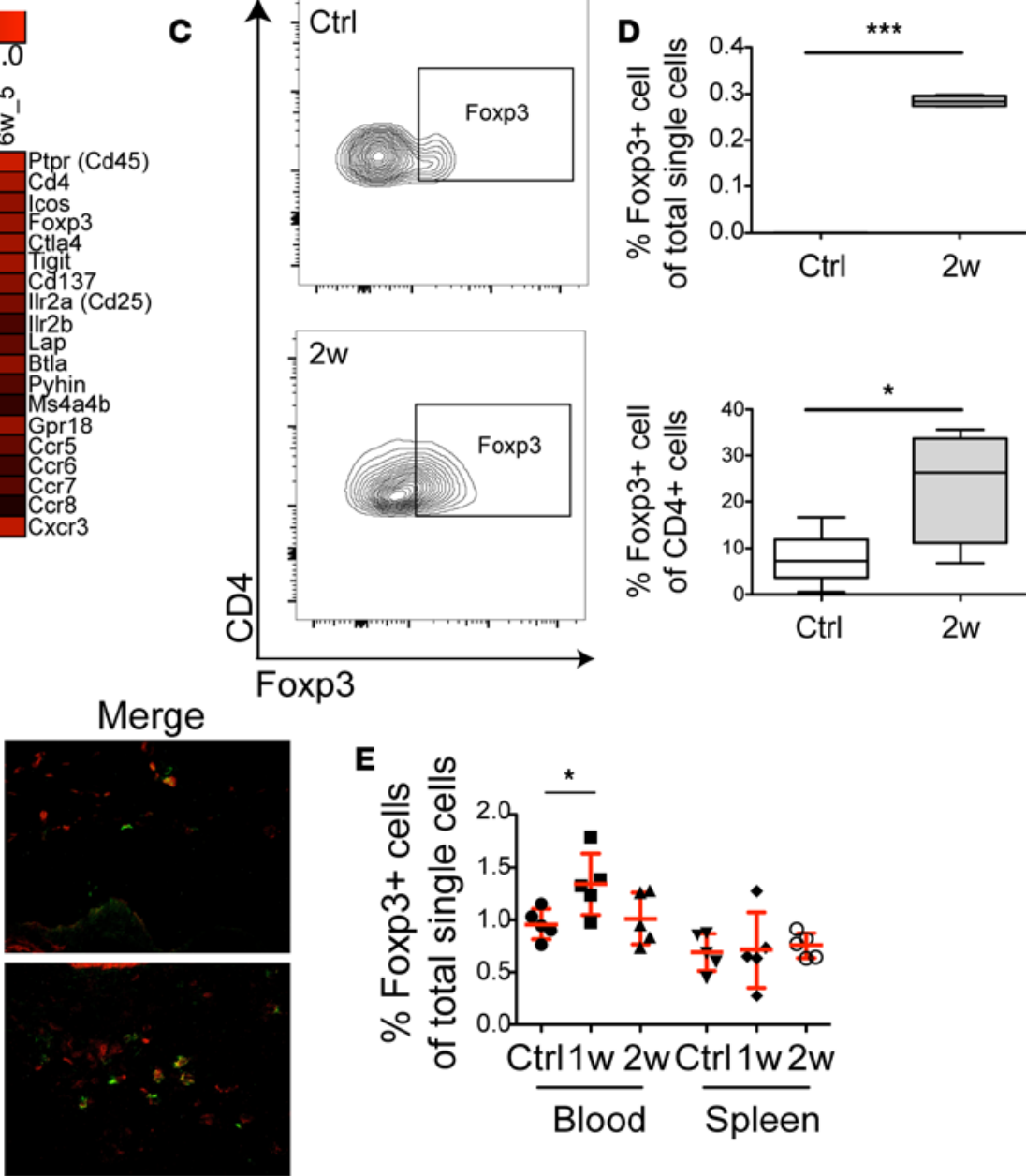

E
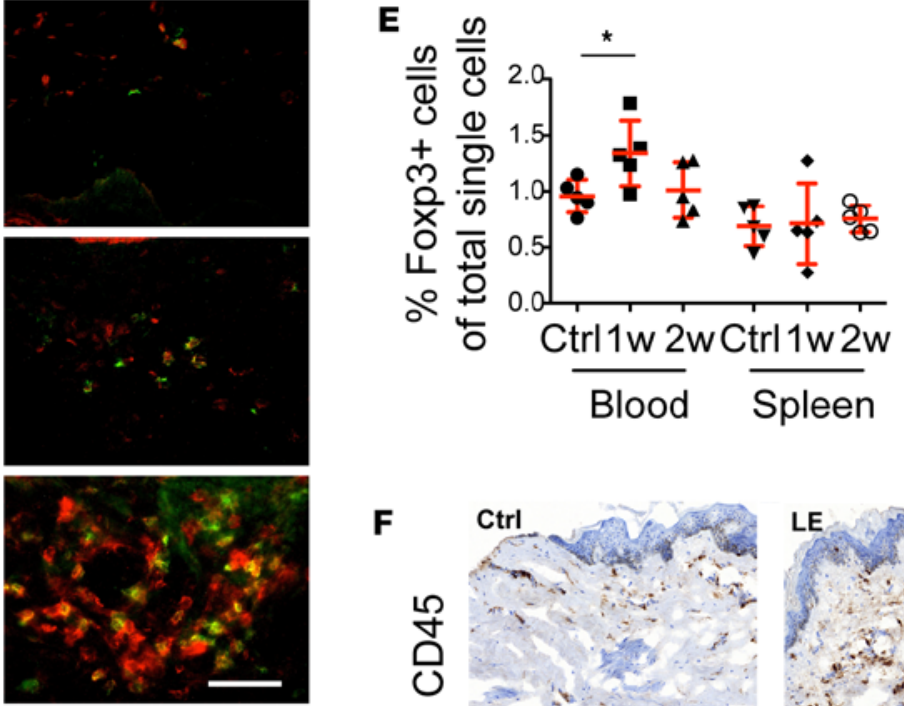

$\mathbf{F}$
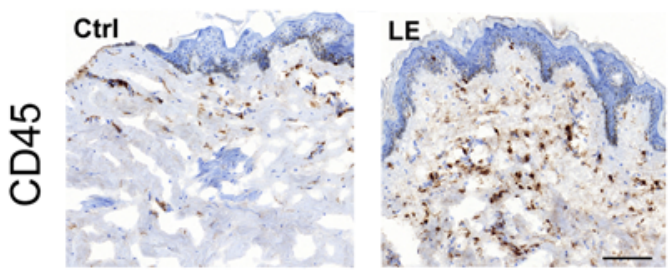

H
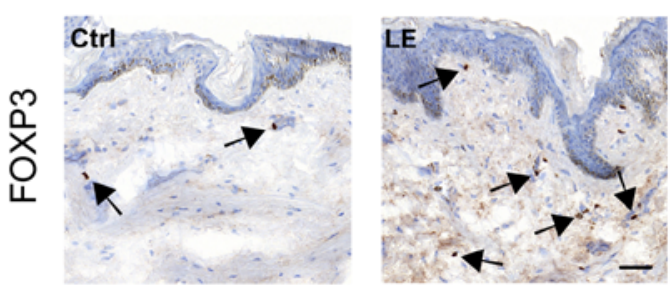

Figure 3. Increased Treg infiltrate in lymphedematous skin in mice and patients. (A) Heat map illustrating upregulation of Treg-associated genes in lymphedematous tissue. The color scale represents log2-fold count changes compared with the mean (indicated as 1:1). (B) Representative immunofluorescence images revealing an increased Treg infiltrate in the lymphedematous skin during lymphedema development 2 and 6 weeks after surgery, in comparison with control unoperated (un-op) mice. Scale bar: $200 \mu \mathrm{m}$. (C) Representative FACS plots of Treg infiltrates in the lymphedematous skin 2 weeks after surgery. Cells were pregated as $C D 45^{+} \mathrm{CD}^{+} \mathrm{CD} 4^{+}$. (D) Quantification of Tregs by FACS, showing an increased Treg percentage both among all single cells and among CD4+ $\mathrm{T}$ cells. (E) Evaluation of Tregs in mouse blood shows an increase in circulating Tregs 1 week after surgery. No changes in Tregs were observed in the spleen ( $n=5$ per group). ( $F$ and $\mathbf{G}$ ) Immunohistochemical staining of patient biopsies from control (Ctrl) and lymphedematous skin (LE) for CD45 $5^{+}$cells indicates a significant increase in inflammatory cells in lymphedema ( $n=5$ per group). Scale bar: $100 \mu \mathrm{m}$. (H and I) Histopathological evaluation of Tregs (arrows) in human lymphedematous skin (LE) indicates an increase in comparison with nonaffected skin (Ctrl) ( $n=5$ per group). Scale bar: $50 \mu \mathrm{m}$. Statistical analysis was done with 1-way ANOVA with Tukey's post-hoc multiple comparison test (E) or Student's $t$ test (D, G, and I), ${ }^{*} P<0.05$, ${ }^{* *} P<0.001$. Each experiment was carried out at least twice. 


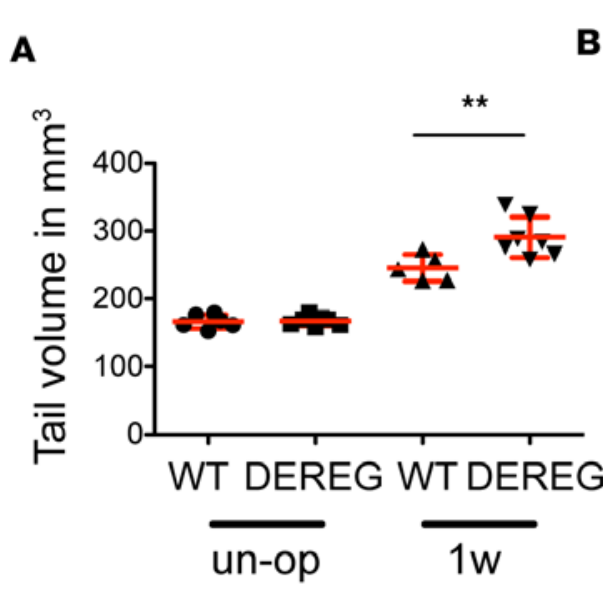

D
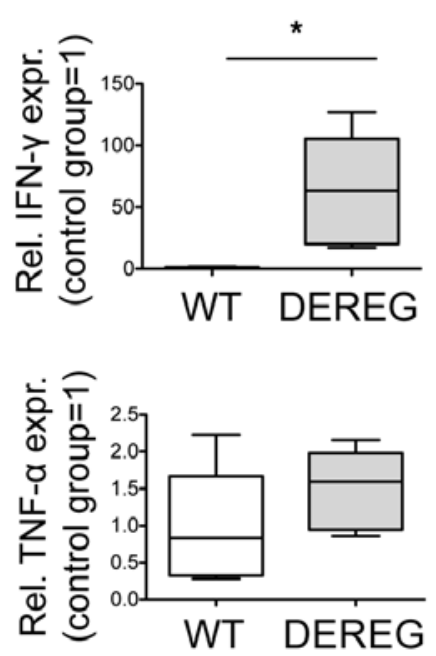

B Lyve-1/Meca-32/Hoechst

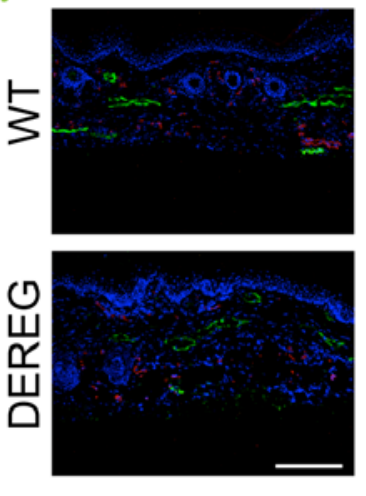

c

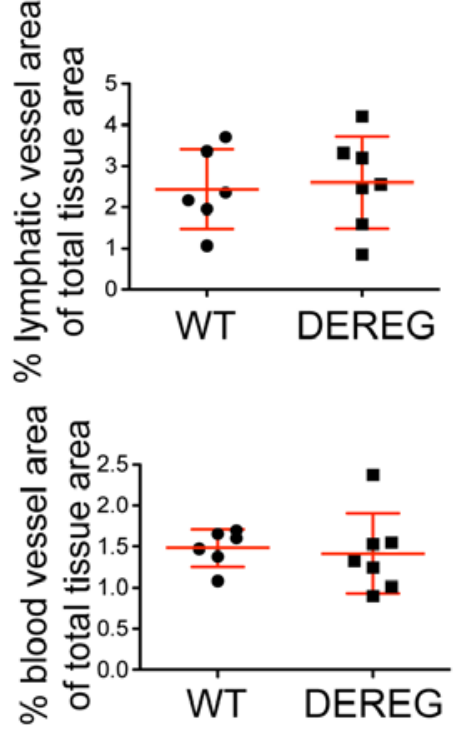

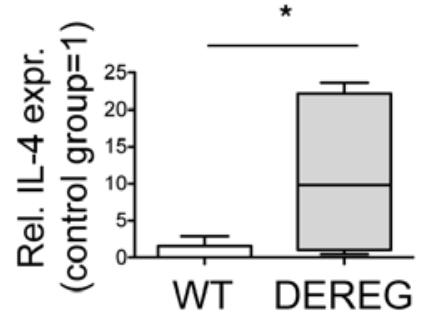
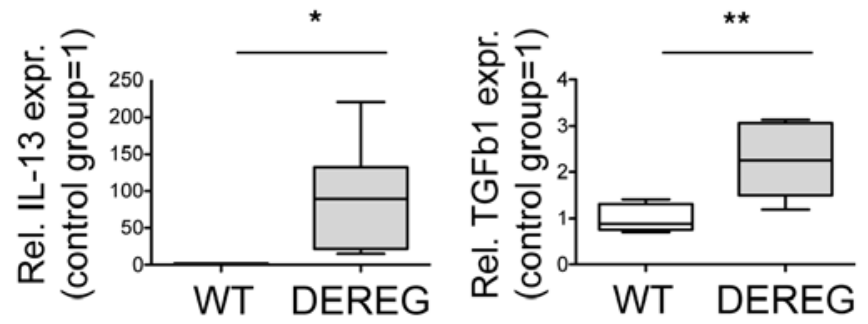

\section{E CD45/Hoechst CD206/Hoechst CD68/Hoechst}
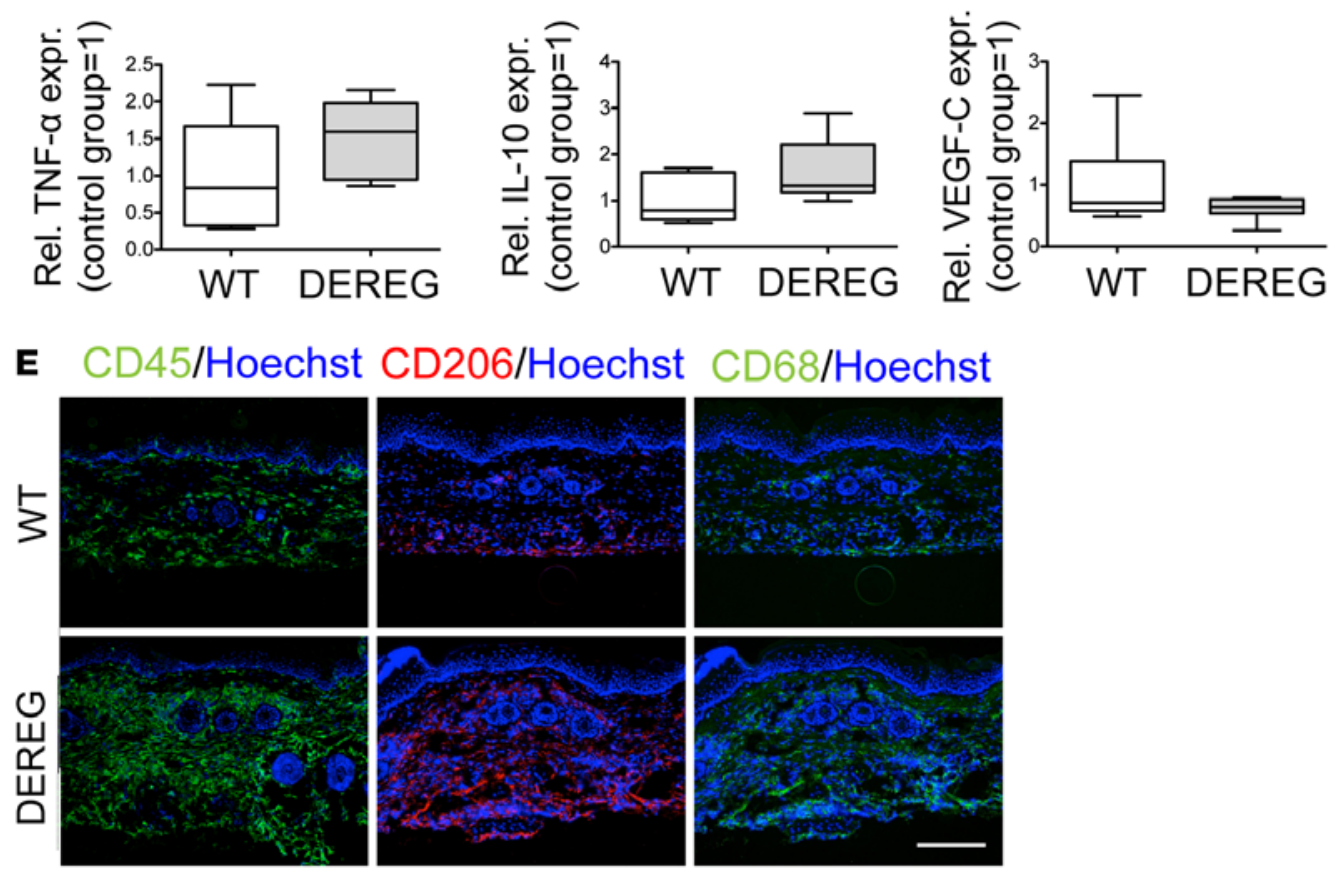

F
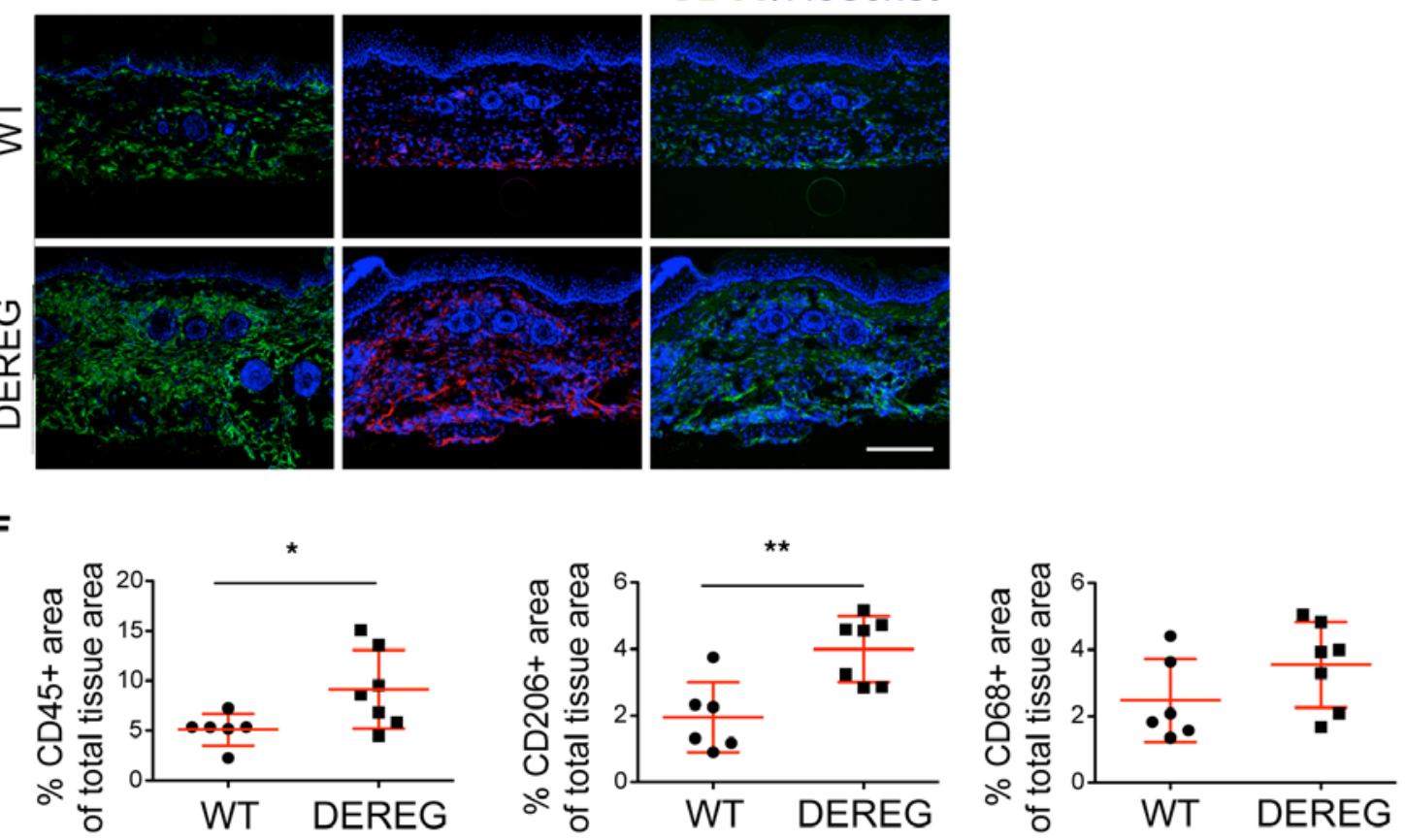
Figure 4. Depletion of Tregs exacerbates lymphedema and tissue inflammation. (A) Evaluation of tail volume upon Treg depletion revealed significantly increased edema in the treated group 1 week after surgery. (B and C) Treg depletion did not evoke any morphological changes in the lymphatic (LYVE-1+) and blood (MECA-32+) vasculature 1 week after surgery. Scale bar: $200 \mu \mathrm{m}$. (D) Expression of inflammatory and antiinflammatory cytokines in tissue extracts of control (WT) and Treg-depleted (DEREG) mice, using RT-PCR, revealed a distinct cytokine milieu. (E and F) Immunofluorescence staining and

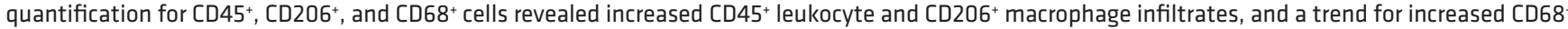
macrophage numbers in the Treg-depleted group. Scale bar: $200 \mu \mathrm{m} . n=5-7$ per group in at least 2 replicates. Statistical analysis was done with 2-way ANOVA with Bonferroni post-hoc test (A) or Student's $t$ test $(\mathbf{C}, \mathbf{D}$, and $\mathbf{F}),{ }^{*} P<0.05,{ }^{*} P<0.01$.

week after surgical lymphedema induction, when measurable edema was detectable. Of note, adoptive Treg transfer significantly reduced tail edema 2 weeks and 3 weeks after surgery ( 1 and 2 weeks after Treg transfer; $P<0.001$; Figure 6, A and B) and the infused Tregs were found to infiltrate the lymphedematous area (Supplemental Figure 2). Assessment of the vascular morphology revealed that lymphatic vessels were smaller and that the tissue area covered by lymphatic vessels was significantly reduced $(P<0.01)$ (Figure 6, C and D) 2 weeks after adoptive Treg transfer, whereas the blood vasculature did not exhibit any morphological changes. A significant reduction in the expression of TGF- $\beta 1, T N F-\alpha$, and $I L-10$ was found in tissue lysates 2 weeks after Treg transfer (Figure 6E), whereas no major change in the expression of VEGF-C was observed. There was a trend towards reduced expression levels of $I F N-\gamma, I L-4$, and $I L-13$ (Figure 6E). The downregulation of inflammatory cytokines was accompanied by reduced infiltration of inflammatory cells, with significantly lower numbers of $\mathrm{CD}^{+} 5^{+}, \mathrm{CD} 206^{+}$, and CD68 ${ }^{+}$cells (Figure 6, F and G).

As adoptive Treg transfer allowed us to investigate fully developed lymphedema 3 weeks after surgery, we were able to evaluate additional parameters related to the severity of the disease. Fibrosis, which is characterized by increased collagen deposition; a hallmark of lymphedema, is associated with increased morbidity and tissue dysfunction, and is also observed in the tail lymphedema model $(5,20)$. The TGF- $\beta$ signaling pathway represents one of the principal signaling axes that promote fibrosis, and TGF- $\beta$ expression was reduced after Treg transfer. Using Sirius Red staining, we found a significantly reduced collagen deposition after Treg transfer $(P<0.001)$ (Figure 7A).

Improved lymphatic vascular function is considered to represent the most critical parameter for successful lymphedema treatment. Thus, we next quantified lymphatic transport from the tail tip to the surgical excision margin by NIR in vivo imaging. There was a significantly increased fluorescence intensity of the lymphatic-specific tracer close to the surgical area in the mice with adoptive Treg transfer $(P<0.01$; Figure 7B and Supplemental Videos 1 and 2), indicating improved lymphatic transport. Taken together, these results reveal that adoptive Treg transfer ameliorated all of the major hallmarks of lymphedema, namely tissue edema, inflammatory cell infiltration, fibrosis, lymphatic vessel enlargement, and impaired lymphatic transport, in a therapeutic setting.

\section{Discussion}

Lymphedema constitutes one of the most common complications following cancer therapy. Despite this, the pathomechanisms underlying disease development are still largely unknown, resulting in the absence of any curative treatments at this time. Importantly, lymphedema may manifest years after the original cancer surgery, and the triggering factors have not been identified, even though it has been thought that inflammatory changes might gradually lead to a decompensation of lymphatic drainage from the affected limb $(4,5)$. In the present study, we have used an established, surgically induced murine lymphedema model and RNA sequencing to identify possible pathomechanisms and therapeutic targets in lymphedema development.

A network analysis of the genes upregulated upon lymphedema development pointed towards an increased presence and/or activation of $\mathrm{T}$ cells, in agreement with previous work by us and others that indicated an increased presence of inflammatory cells during lymphedema development $(5,7)$ and suggested a particular pathogenic role for $\mathrm{CD}^{+}$cells $(6,8,21)$. Consistent with these studies, depletion of the $\mathrm{CD}^{+}$cell population by systemic application of a blocking antibody resulted in lymphedema amelioration with improved lymphatic vascular function, indicating a major functional role of $\mathrm{CD}^{+} \mathrm{T}$ cells in the pathogenesis of lymphedema.

$\mathrm{CD}^{+} \mathrm{T}$ cells encompass multiple subsets, including $\mathrm{T}_{\mathrm{H}} 1, \mathrm{~T}_{\mathrm{H}} 2$, and Tregs. An important new finding of our RNA sequencing study was that Foxp3, a marker gene for Tregs, was among the most highly upregulated genes in lymphedema, together with several other Treg-associated genes. Indeed, the increased mRNA expression levels in total lymphedema skin extracts were associated with increased numbers of Tregs infiltrating lymphedematous skin in the experimental mouse tail model. Importantly, increased numbers of 
A

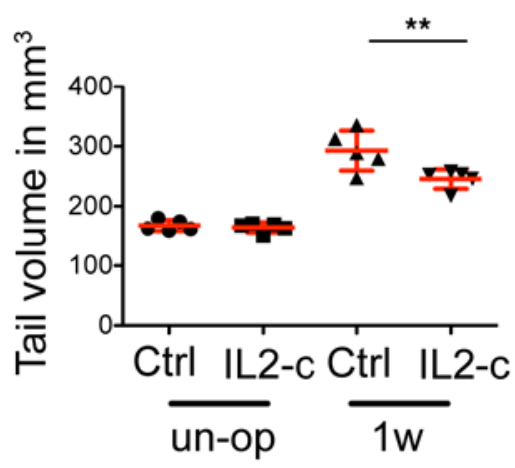

B

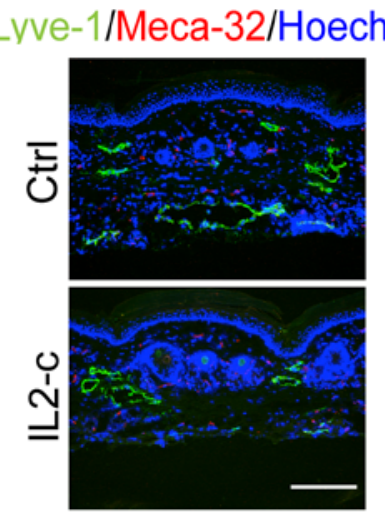

\section{CD45/Hoechst CD206/Hoechst CD68/Hoechst}
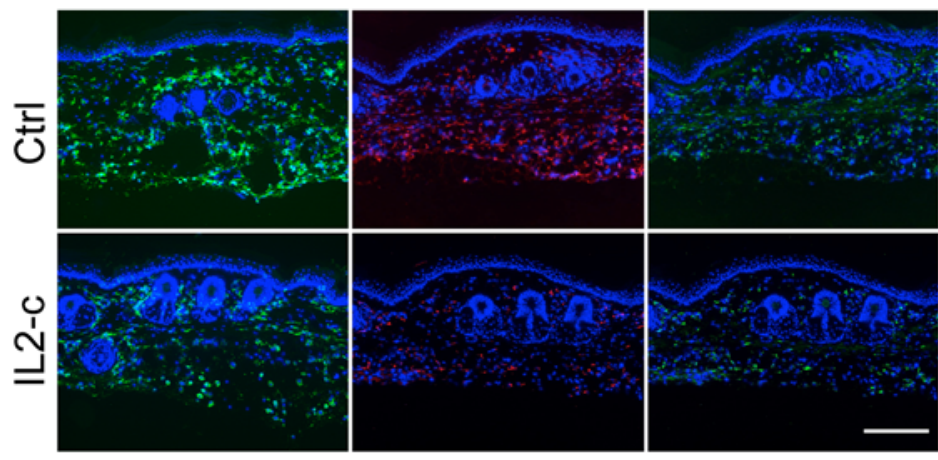

E
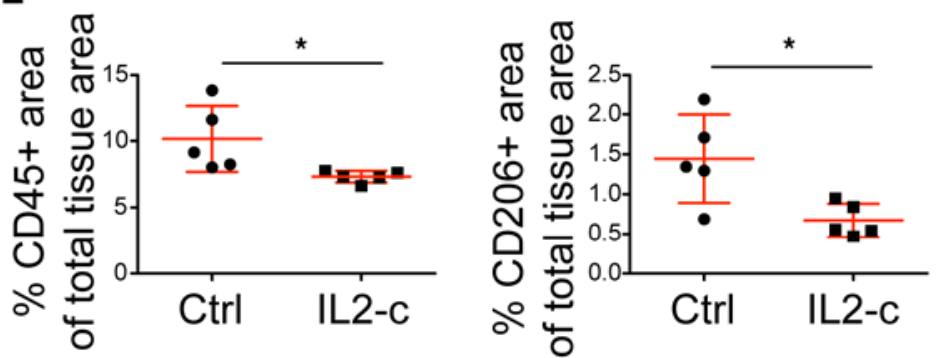

C
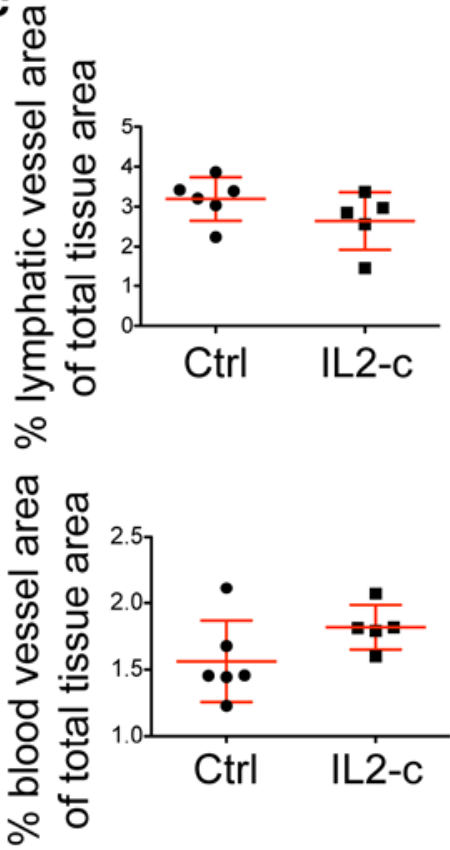

Figure 5. Systemic expansion of Tregs by IL-2/anti-IL-2 mAb complex (IL2-c) reduces tail edema. (A) Tail volume evaluation upon Treg expansion in lymphedema revealed significantly decreased edema in the IL2-c-treated group compared with controls (Ctrl) 1 week after surgery. (B) Representative images of lymphatic (Lyve-1+) and blood (Meca-32+) vessels 1 week after surgery. Scale bar: $200 \mu \mathrm{m}$. (C) Quantification of lymphatic and blood vascular area did not reveal any changes 1 week after surgery. (D) Representative immunofluorescence staining for CD45', CD206', and CD68 ${ }^{+}$cell infiltrate after IL2-C treatment. Scale bar: $200 \mu \mathrm{m}$. (E) Quantification of the immunofluorescence staining for $\mathrm{CD}^{2} 5^{+}, \mathrm{CD}_{206}{ }^{+}$cells, and CD68 $8^{+}$macrophages indicates reduced inflammation and a decrease in all examined immune cell populations after 1 week of treatment. $n=5-6$ per group, in at least 2 replicates. Statistical analysis was done with 2-way ANOVA with Bonferroni post-hoc test (A) or Student's $t$ test (C and $\mathbf{E}),{ }^{*} P<0.05,{ }^{* *} P<0.01$.

Tregs were also found in the affected skin of human lymphedema patients. To the best of our knowledge, this is the first demonstration of increased Treg numbers in human lymphedema.

Apart from lymphoid tissues, small numbers of Tregs have also been found to reside within nonlymphoid healthy tissues (22), increasing in numbers in response to inflammatory stimuli. Infiltration of Tregs modulates tissue-specific inflammatory responses, e.g., in inflammatory bowel disorder (23) and muscular dystrophy (24). The localization of Tregs in the lymphedematous skin indicated a potential role in suppressing tissue inflammation. Previous work in which $\mathrm{CD} 25^{+}$cells were systemically depleted using antibodies did not report a major effect on lymphedema development, likely owing to the collateral depletion of both activated $\mathrm{CD} 4^{+}$cells and Tregs (8). In contrast, specific and targeted depletion of Tregs by application of diphtheria toxin to DEREG mice exacerbated lymphedema development, leading to an increased inflammatory infiltrate and a distinct cytokine profile, with elevated 

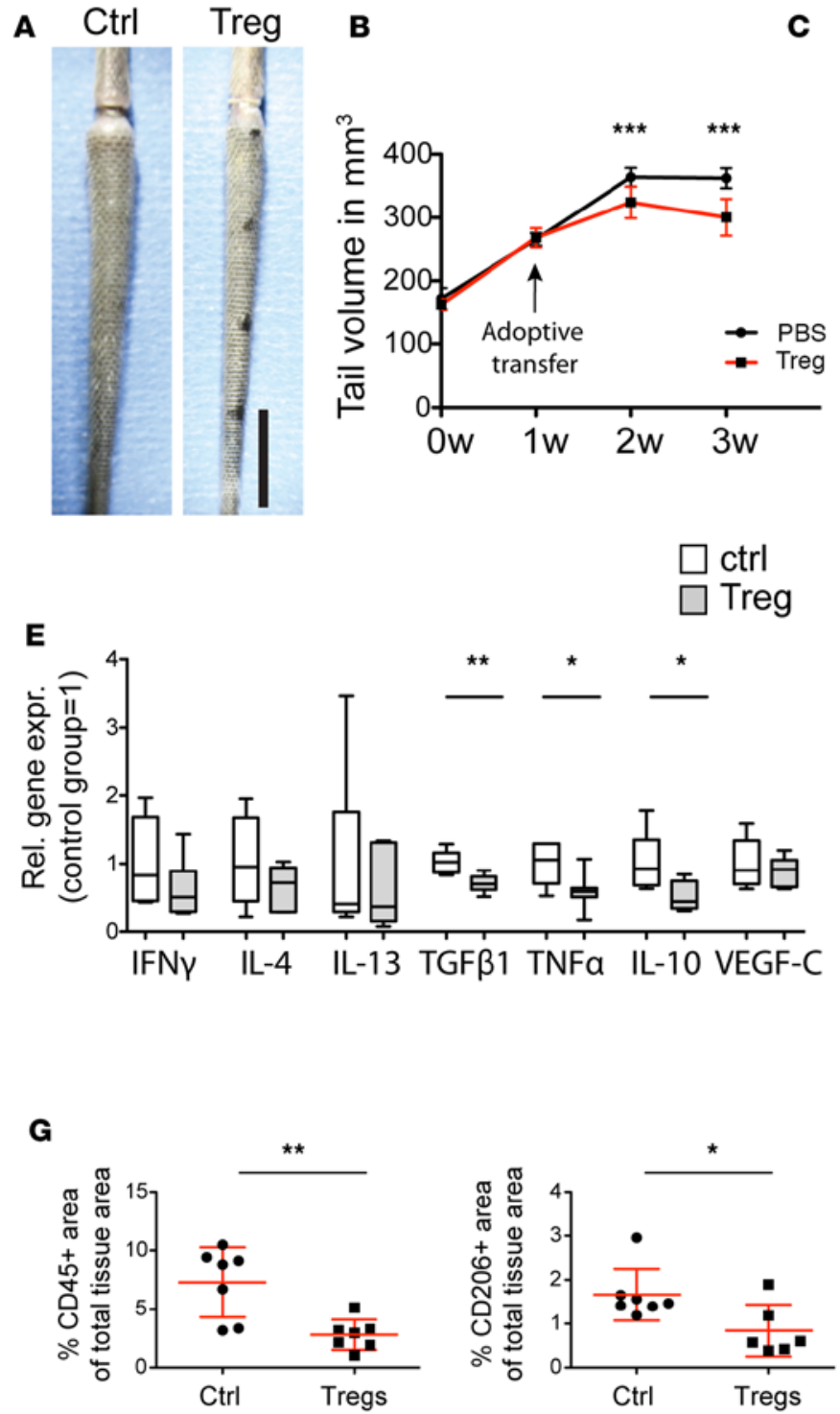

C

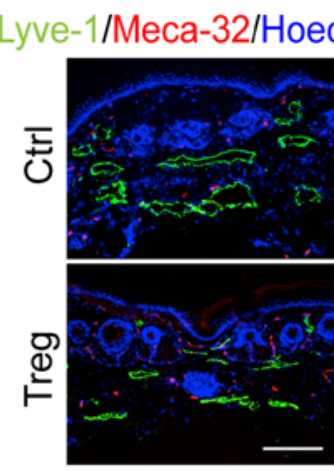

ctrl

$\mathbf{F}$
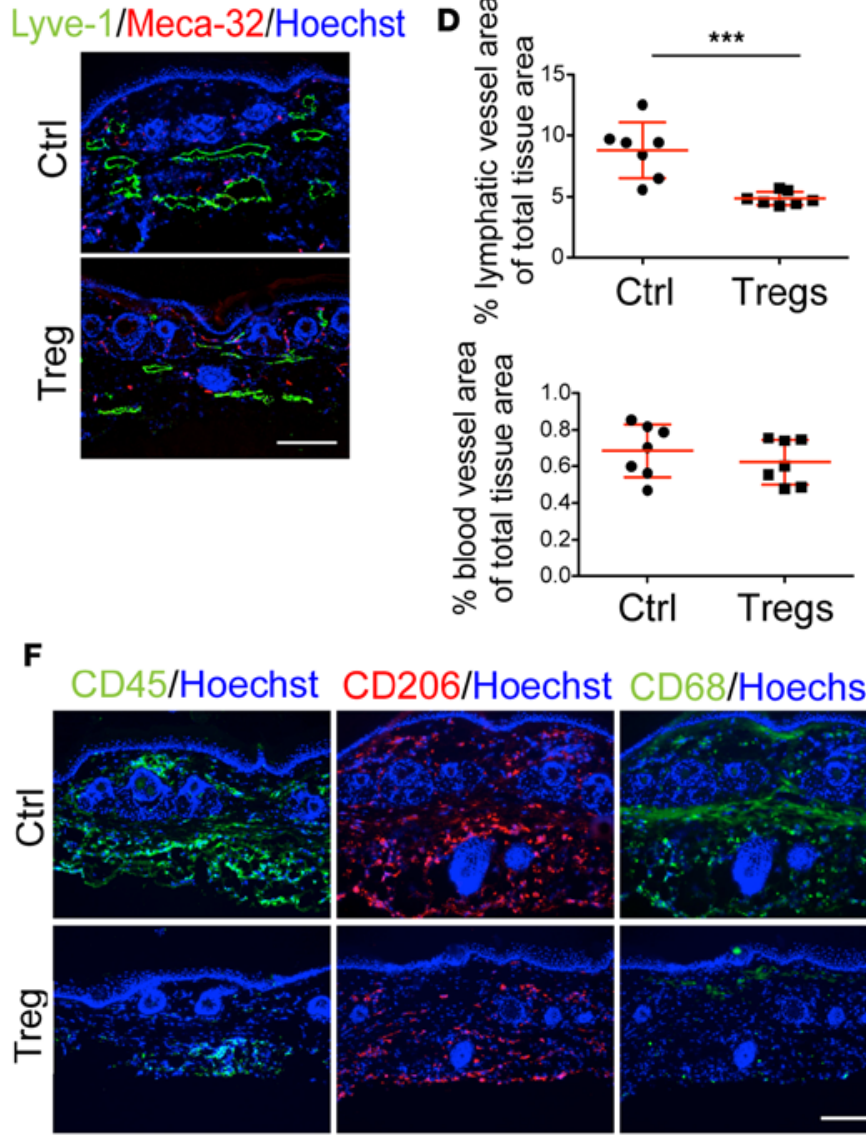

CD68/Hoechst
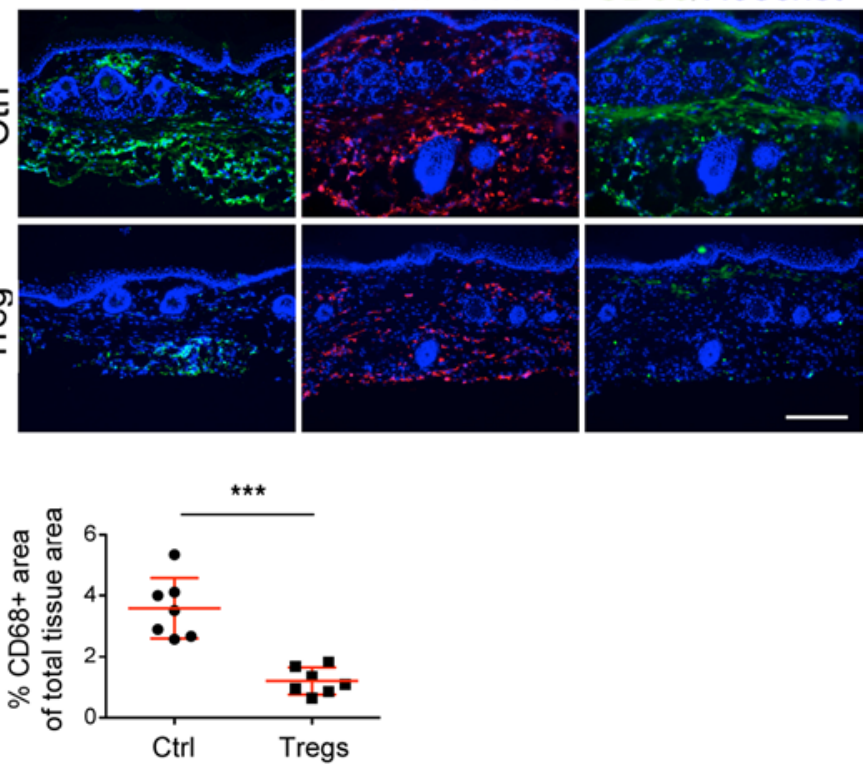

Figure 6. Therapeutic adoptive transfer of Tregs reduces inflammation and ameliorates lymphedema. (A) Representative images of tails of control (Ctrl) and Treg-treated mice 3 weeks after surgery ( 2 weeks after the treatment). Scale bar: $1 \mathrm{~cm}$. (B) Tail volume evaluation. Treg transfer was performed 1 week after surgery. Adoptive transfer of Tregs led to significant reduction of edema 1 and 2 weeks after treatment ( 2 and 3 weeks after surgery). (C) Representative immunofluorescence staining for lymphatic (Lyve-1+) and blood (Meca-32+) vessels 3 weeks after surgery. Scale bar: $200 \mu \mathrm{m}$. (D) Image analysis of the lymphatic and blood vasculature revealed significant morphological changes with reduced lymphatic vascular expansion in the Treg-treated group, whereas blood vessels remained unchanged. (E) Expression profiling of inflammatory cytokines by qPCR showed a trend for reduced expression of $\mathrm{T}_{H} 1$ - and $\mathrm{T}_{\mathrm{H}} 2$-associated cytokines. Expression of TCF- $\beta 1$, TNF- $\alpha$, and IL-10 was significantly reduced. (F and $\left.\mathbf{G}\right)$ Immunofluorescence staining for $C D 45^{+}, \mathrm{CD}_{206^{+}}$, and $\mathrm{CD}^{+} 8^{+}$cells showed a marked overall decrease in inflammation 2 weeks after Treg transfer, with a significant reduction of all 3 cell populations analyzed. Scale bar: $200 \mu \mathrm{m} . n=5-7$ per group, replicated once. Statistical analysis was done with 2-way ANOVA with Bonferroni post-hoc test (B) or Student's $t$ test (D, E, and G), ${ }^{*} P<0.05,{ }^{* *} P<0.01,{ }^{* * *} P<0.001$.

levels of $I F N-\gamma, I L-13, I L-4$, and $T G F-\beta 1$ that have been reported to impair lymphatic vessel function $(12,14,15)$. A disadvantage of this model is the severe autoimmunity following prolonged Treg depletion (25); thus, time points later than 1 week could not be analyzed.

$T G F-\beta 1$ is a profibrotic factor that has been reported to promote lymphedema progression (15). Similarly, $\mathrm{T}_{\mathrm{H}} 2$ responses, characterized by the expression of the $I L-13$ and $I L-4$ cytokines, have previously been found to modulate soft-tissue fibrosis during lymphedema development, and blockade of $I L-4$ and $I L-13$ attenuated lymphedema and improved fibrosis and lymphatic function (21). The effects of $\mathrm{T}_{\mathrm{H}} 2$ responses are likely not restricted to fibrosis development since a recent study found that $\mathrm{T}_{\mathrm{H}} 2$ cytokines negatively 
A
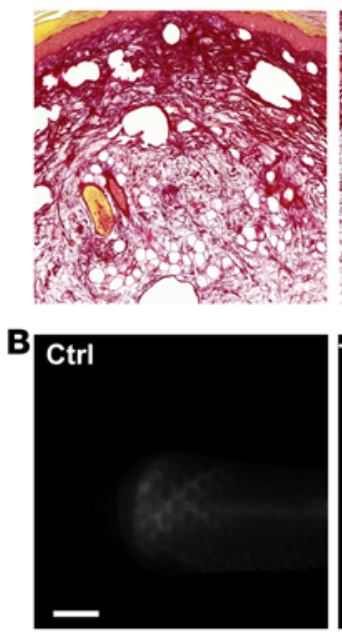

Ctrl

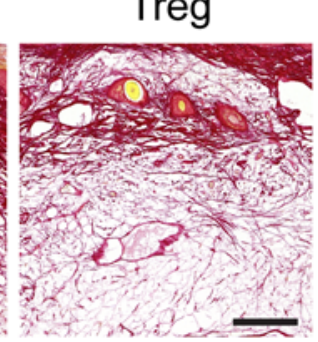

Treg
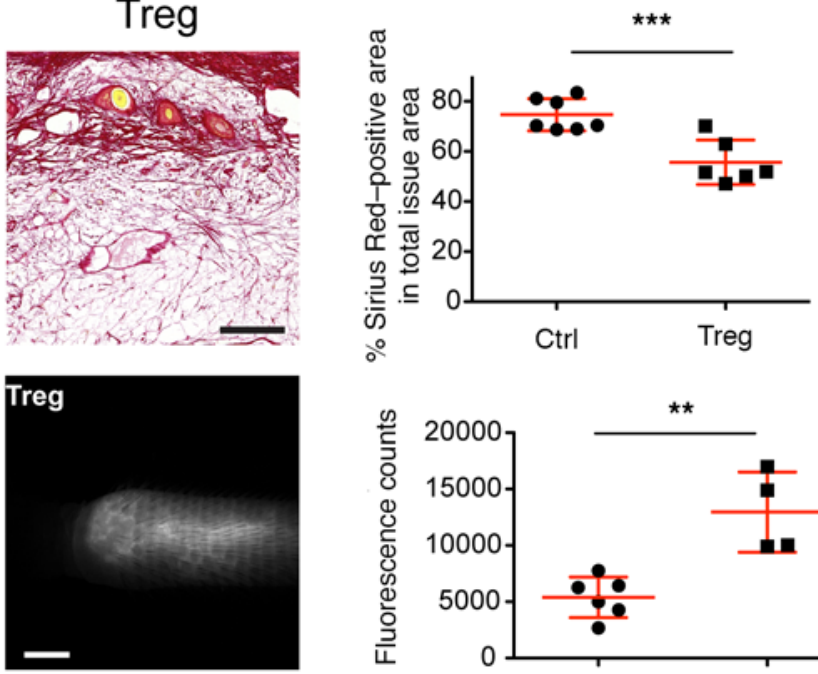

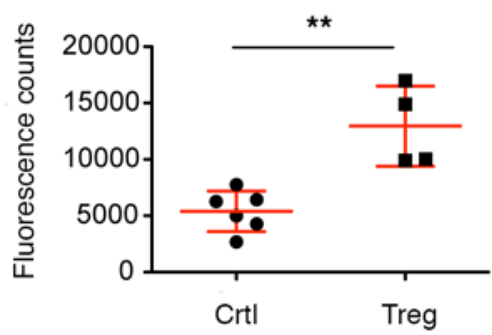

Figure 7. Therapeutic Treg transfer reduces fibrotic tissue deposition and improves lymphatic vessel function. (A) Sirius Red histological staining to evaluate fibrotic tissue deposition in the control (Ctrl) and treated (Treg) group revealed a significant reduction of fibrosis 2 weeks after Treg transfer. Scale bar: $200 \mu \mathrm{m}$. (B) Assessment of lymphatic vessel function using noninvasive near-infrared imaging showed marked improvement of the lymphatic transport capacity after Treg treatment, as indicated by the increased amount of tracer reaching the surgical excision site 20 minutes after infusion near the tip of the tail. Scale bars: $2 \mathrm{~mm} . n=4-6$, replicated once. Statistical analysis was performed with Student's $t$ test, ${ }^{* *} P<0.01,{ }^{* *} P<0.001$.

affect lymphatic vessel formation and function both in vitro and in vivo (14). In addition, IFN- $\gamma$, which typically characterizes $\mathrm{T}_{\mathrm{H}} 1$ immune responses, suppressed the expression of lymphatic-specific genes and reduced lymphangiogenesis, promoting lymphatic vessel regression (12). Collectively, the expression of $T G F-\beta 1$ and the upregulation of $I L-4, I L-13$, and $I F N-\gamma$ upon Treg ablation are likely to exacerbate fibrosis and presumably lymphatic vascular dysfunction, which together aggravate lymphedema development. Thus, our finding that these cytokines were significantly upregulated after Treg depletion supports a potential role for Tregs in suppressing lymphedema-associated fibrosis.

Apart from their capability to suppress $\mathrm{T}$ cell responses, Tregs strongly impact myeloid cell populations in their vicinity as well, hindering the function of neutrophils and proinflammatory macrophages (26). Consequently, manipulating Tregs has the potential to control the pathology of a wide spectrum of inflammation-associated diseases. Application of IL2-c in naive mice has been found to rapidly and selectively expand Tregs $(19,27)$ that possess superior suppressive functions, thereby successfully inducing resistance to autoimmune encephalomyelitis and promoting long-term acceptance of islet allografts (28). Our findings that IL2-c treatment resulted in reduced swelling, and prevented inflammation and macrophage infiltration, are thus consistent with a role for Tregs in limiting lymphedema.

IL2-c treatment is likely not suitable for clinical application in lymphedema in a therapeutic setting, owing to potential side effects on activated effector $\mathrm{T}$ cells, even though 2 recent clinical studies have demonstrated beneficial results of low-dosage IL-2 treatment in graft-versus-host disease (29) and autoimmune vasculitis patients (30). However, the promising results of the IL2-c application in lymphedema prevention triggered us to evaluate the therapeutic effect of Tregs in a setting that could be translatable into clinical application (31). Adoptive transfer of autologous or donor-derived Tregs represents a promising immunotherapeutic strategy, in which Tregs are isolated, enriched, expanded, and reinfused into the recipient patient (32). Treg transfer was found to be successful in a number of experimental disease models and exhibited encouraging results in several clinical trials by inducing allograft tolerance $(33,34)$. In the present study, we found that therapeutic Treg transfer at 1 week after lymphedema-inducing surgery indeed led to a strong amelioration of lymphedema development for up to 2 weeks of observation, concomitant with a trend towards reduced expression of $\mathrm{T}_{\mathrm{H}} 1$ and $\mathrm{T}_{\mathrm{H}} 2$ cytokines and reduced levels of $T N F-\alpha$, a proinflammatory cytokine known to drive myeloid cell activation as well as blood and lymphatic vascular remodeling (35). 
Importantly, adoptive Treg transfer strongly reduced fibrosis, a hallmark of lymphedema. The decreased expression levels of TGF- $\beta 1$ corroborate this concept, confirmed by the decreased collagen deposition, and the observed reduced expression of $I L-10$ might also relate to fibrosis abrogation besides reduction of inflammation (36). In addition, Tregs might directly contribute to tissue repair (37), as was found during muscle regeneration and mucosal barrier repair in the colon (37-39). Lymphatic vascular dysfunction is considered to represent the primary cause of lymphedema. Thus, it is important to note that Treg transfer potently rescued lymphatic function, improving the transport of a lymphatic-specific tracer from the tail tip to the edge of the surgical excision margin in lymphedematous tails, likely via reduction of the levels of negative lymphatic mediators and by limiting tissue fibrosis.

One should keep in mind that Tregs might also directly reduce tissue inflammation, thereby contributing to lymphedema amelioration by decreasing the inflammatory burden, indicating that antiinflammatory treatment might be beneficial in the treatment of lymphedema. Indeed, previous work indicates that application of a nonsteroidal antiinflammatory drug (ketoprofen) improves edema in mice (40), although without major effects on lymphatic vessel morphology or function. Thus, antiinflammatory treatment alone might not suffice to fully rescue lymphatic impairment, and direct tissue repair mechanisms, such as those mediated by Tregs, might be needed in addition. Further research is needed to elucidate the detailed mechanisms by which Tregs exert their beneficial action in lymphedema.

Collectively, our results identify, to the best of our knowledge for the first time, a distinct mechanistic role for Tregs in lymphedema, in which yet unidentified signals arising from inflamed lymphedematous tissue recruit Tregs to suppress the $\mathrm{T}_{\mathrm{H}} 1 / \mathrm{T}_{\mathrm{H}} 2$ immune response and tissue fibrosis, leading to improved lymphatic function. Thus, therapeutic intervention by Treg transfer may reverse the pathological manifestations of the disease, at least during early phases of lymphedema development. The potential application of this therapy to human patients necessitates careful consideration though, since lymphedema is often a postcancer complication and immunomodulation by Treg transfer may raise undesired complications (e.g., reduced antitumor immune responses) in this group of patients. Potential ex vivo manipulation of Treg homing properties prior to infusion might be an option to overcome this problem, by targeting Tregs specifically to the skin for example and minimizing systemic side effects (41-43). Despite possible challenges in translating these findings into a treatment, we suggest Tregs as a potential novel therapeutic approach for lymphedema, a disease with currently no curative therapeutic approach.

\section{Methods}

Surgical induction of lymphedema. To investigate the pathologic processes underlying lymphedema as well as potential approaches to target those, a well-established surgically induced mouse tail lymphedema model was employed as previously described (5). Briefly, a circumferential portion of skin was excised $2 \mathrm{~cm}$ distal to the tail base. The collecting lymphatic vessels were identified by Evans Blue injection and ablated. Lymphedema developed within 1 week and remained for up to 6 weeks. Lymphedematous tail tissue was harvested from mice 2 and 6 weeks after surgery and together with control (unoperated) tissue was processed for RNA sequencing analysis ( $n=5$ per group per time point). All animal studies were performed on 8- to 12-week-old C57BL/6J female mice (Janvier Labs) or DEREG mice (provided by Tim Sparwasser, Institute of Infection Immunology, TWINCORE, Hannover, Germany), with wild-type littermates serving as controls.

$R N A$ sequencing. Total RNA samples $(1 \mu \mathrm{g})$ were depleted of ribosomal RNA and then reverse transcribed into double-stranded cDNA, using the TruSeq Stranded mRNA Sample Prep Kit (Illumina). cDNA samples were fragmented, end repaired, and polyadenylated before ligation of TruSeq adapters. Following selective enrichment of adapter-ligated fragments with PCR, quality and quantity of the libraries was assessed with a Qubit Fluorometer (Life Technologies) and a Bioanalyzer 2100 (Agilent Technologies). Libraries $(10 \mathrm{nM})$ in Tris- $\mathrm{Cl} 10 \mathrm{mM}, \mathrm{pH} 8.5$ with $0.1 \%$ Tween 20 were used for cluster generation with the TruSeq SR or PE Cluster Kita v3-cBot-HS (Illumina). Sequencing was performed using the Illumina HiSeq2500 to generate single-end reads of $101 \mathrm{nt}$ in length. The raw reads were first purified by removing adapter sequences, trimming low-quality ends, and filtering reads with low quality (phred quality $<20$ ) using Trimmomatic (44). High-quality reads were then aligned to the Mus musculus reference genome (build GRCm38) and gene expression levels were quantified using RSEM (Version 1.2.12) (45). DESeq2 was used to detect differentially expressed genes (R version: 3.1.0, DEseq2 version: 1.4.5) (46), using an exact test adapted for overdispersed data, and a $P$ value of less than 0.05 was considered significant (Benjamini and Hochberg method). Further analysis of the gene networks overrepresented in the differentially expressed 
genes was performed using MetaCore (Thomson Reuters). The raw RNA sequencing data can be found in the EMBL-EBI repository with the accession number PRJEB15150.

Human tissue samples. To evaluate the clinical relevance of the experimental findings, human specimens were obtained from the Department of Plastic Surgery of the Aristotle University of Thessaloniki from breast cancer patients undergoing autologous lymph node transfer or breast reconstruction after breast cancer treatment. Five lymphedema and 5 control patients were included (all female). The lymphedema patients had a measurable degree of lymphedema (grade I-II) after breast cancer treatment that included mastectomy or lobectomy with chemotherapy and radiotherapy. The affected limb preoperatively underwent lymphatic massage to reduce fluid. Skin samples were obtained from the distal tail of Spence, close to the axilla. Control biopsies were obtained from the abdominal or dorsal skin either from lymphedema patients $(n=3)$ (treated with mastectomy and radiotherapy/chemotherapy) or patients that did not have signs of lymphedema $(n=2)$. Due to the small patient number, we did not attempt to match patients by age, BMI, or other demographic parameters.

Human skin immunohistochemistry. Immunohistochemical stainings of human skin biopsies were performed using an automated Ventana Benchmark Ultra. The following antibodies were used: mouse antihuman CD45 (Dako, M0701, clone 2B11, 1:250, antigen retrieval with Tris-Borate buffer for 24 minutes); mouse anti-human FOXP3 (Abcam, ab20034, clone 236A/E7, 1:50, antigen retrieval with Tris-Borate buffer for 92 minutes; for the costaining, samples were not pretreated and the Ventana UltraView Universal Antibody Kit was used); and mouse anti-human CD25 (Novocastra, NCL-CD25-305, clone 4C9, 1:50, with the Leica Bond Refine Detection Kit and no pretreatment).

$R N A$ extraction and RT-PCR. RNA was extracted from a 2-3 mm full-thickness tail skin portion, 1-2 $\mathrm{cm}$ distal to the surgical excision margin, 2 and 6 weeks after surgery or from the same tail area of unoperated mice ( $n=5$ per group), using TRIzol (Ambion) after mechanical homogenization in a TissueLyser (QIAGEN), followed by purification with the NucleoSpin RNA Kit according to the manufacturer's protocol (Macherey-Nagel). For RNA sequencing, evaluation of RNA integrity was performed using the Agilent RNA 6000 Nano kit (Agilent Technologies). cDNA was transcribed from $1 \mu \mathrm{g}$ RNA, using the High Capacity Reverse Transcription kit (Applied Biosystems). FastStart SYBR Green master mix (Roche) and a 7900HT Fast Real-Time PCR System (Applied Biosystems) were used for PCR reactions. Expression values were normalized to RPLP0 (36B4) and fold changes in gene expression were calculated using the $\Delta \Delta_{\mathrm{CT}}$ method. The primer sequences used are provided in Supplemental Table 1.

Mouse histology. Immunofluorescence staining was performed on frozen sections as described (5). For Treg staining, 7- $\mu \mathrm{m}$ frozen sections obtained from unoperated and operated DEREG mice were fixed in acetone/methanol and incubated for 2 hours at room temperature with chicken anti-GFP (1:1,000, GFP1010, Aves) in antibody diluent (Zytomed Systems), followed by secondary antibodies for 30 minutes at room temperature (donkey anti-chicken Alexa 488, 1:200, catalog 703.545.155, Jackson ImmunoResearch Laboratories). Subsequently, tissues were stained for CD4 (biotinylated rat anti-CD4, 1:50, clone H129.19, BD Pharmingen), followed by Alexa 594-conjugated streptavidin (1:500, catalog S32356, Life Technologies) and Hoechst 33342 (1:1,000). For all other immunofluorescence stainings, tissues were fixed and incubated for 2 hours with blocking solution (5\% nonimmune donkey serum, 0.2\% BSA, 0.3\% Triton X-100 in PBS) and incubated overnight $\left(4^{\circ} \mathrm{C}\right)$ with antibodies against CD45 (AF114, R\&D Systems, 1:100), CD68 (clone FA-11, Abcam, 1:200), or CD206 (AF2535, R\&D Systems, 1:200). The sections were subsequently incubated for 30 minutes with Alexa 488- or Alexa 594-conjugated secondary antibodies (all 1:200, catalog A-21208, A-11058, and A-11055) and Hoechst 33342 (1:1,000) (all from Life Technologies). Five images per tissue section were taken with an Axioskop 2 mot plus microscope (Carl Zeiss) equipped with an AxioCam MRc camera and a Plan Apochromat 10×/0.45 NA or Plan Neofluar 40×/0.75 NA objective. Image quantification was performed using ImageJ $(\mathrm{NIH})$ in a blinded fashion. For the quantification of the lymphatic area, lymphatic structures including the luminal space were used.

For histological analysis of fibrosis, 2- to 3-mm-wide tail pieces were dissected distal to the excision area and fixed in 4\% PFA. The tissue was decalcified for 10 days in 0.5 M EDTA (Sigma-Aldrich) and then embedded in paraffin. Sections $(6 \mu \mathrm{m})$ were obtained and stained with Picrosirius Red solution (Sigma-Aldrich) for 1 hour and then dipped in acidified water $(0.5 \%$ acetic acid in distilled water). Images were acquired using a Slide Scanner (Panoramic 250 BF Flash, 3DHISTECH) equipped with a Brightfield CIS 3CCD VCC-F52U$25 \mathrm{CL}$ camera and a $20 \times / 0.8$ NA objective. For collagen quantification, ImageJ was used.

Flow cytometry. Single-cell suspensions of spleen and blood were stained for surface markers followed by intracellular staining for Foxp3 using a Foxp3/Transcription Factor Staining Set (eBioscience) accord- 
ing to the manufacturer's guidelines. For the analysis of lymphedematous skin, full-thickness tail skin was obtained distal to the surgical margin and single-cell suspensions were generated as described (5). Briefly, the tissue was minced and incubated in collagenase II $(5 \mathrm{mg} / \mathrm{ml})$ and DNase I $(0.05 \mathrm{mg} / \mathrm{ml})$ (both from Sigma-Aldrich) in RPMI medium (Gibco) for 30 minutes at $37^{\circ} \mathrm{C}$ on a shaker. Single-cell suspensions were filtered through $70-\mu \mathrm{m}$ and $40-\mu \mathrm{m}$ cell strainers and resuspended in FACS buffer ( $2 \%$ FBS, 2 mM EDTA in PBS). The following antibodies were used: CD16/CD32 (1:100, clone 93); CD45 (Pacific blue, 1:400, clone 30-F11); CD3 (PECy7, clone 145-2C11, 1:200); CD4 (APC, clone GK1.5, 1:200) (all from BioLegend); and Foxp3 (PE, clone FJK-16s, 1:100, eBioscience). Fixable Aqua Zombie (1:300, catalog L349957, Life Technologies) was used for live/dead discrimination.

Depletion of $\mathrm{CD}^{+} \mathrm{T}$ cells. Experimental animals were depleted of $\mathrm{CD} 4^{+}$cells using a monoclonal neutralizing antibody against CD4 (clone GK1.5, Bio X Cell). The antibody was administered at a dose of 10 $\mu \mathrm{g} / \mathrm{g}$ per mouse i.p. on the day of operation, the day following, and thereafter once every 5 days for a total of 4 weeks, resulting in a more than $98 \%$ depletion of $\mathrm{CD}^{+}$cells in the blood. Control animals were treated with nonspecific IgG isotype control antibodies i.p. at the same dose and time points.

In vivo Treg ablation. To specifically deplete Tregs, DEREG mice generated as previously described on the C57BL/6J background were used (25). One microgram of diphtheria toxin (Calbiochem) was administered i.p. to the mice 1 day prior to the surgery, the day of the surgery, and afterwards every third day for 1 week, leading to an approximately $80 \%$ reduction in the number of Tregs in blood. No signs of overt morbidity were observed within this time frame.

In vivo Treg expansion. For the expansion of Tregs, IL2-c were used as previously published (19). Mice were injected for 3 consecutive days i.p. with $3 \mu \mathrm{g}$ of IL-2 (catalog 34-8021-85, eBioscience) that had been preincubated for 15 minutes on ice with $15 \mu \mathrm{g}$ of anti-IL-2 mAb (clone JES6-1, Bio X Cell), leading to a more than 4-fold increase in the number of Tregs in the blood. The mice underwent surgery the day following the last administration and were euthanized 1 week later.

Adoptive Treg transfer. Adoptive Treg transfer was performed 1 week after the surgery to induce lymphedema. Tregs were expanded in DEREG mice using IL2-c as described above. Two days after the last injection, mice were euthanized and the spleens were removed and passed through a $40-\mu \mathrm{m}$ strainer to create single-cell suspensions. Cells were prepurified using the $\mathrm{CD} 4{ }^{+} \mathrm{T}$ Cell Isolation Kit (Miltenyi Biotec), followed by staining for CD4 (clone GK1.5, APC, 1:200, BioLegend) and CD25 (clone PC61, PerCP, 1:100, BioLegend). Tregs were sorted as $\mathrm{CD}^{+} \mathrm{GFP}^{+} \mathrm{CD} 25^{+}$cells using a BD FACS Aria Ilu (BD Biosciences). Approximately 0.8-0.9 million cells in PBS were injected into the tail vein close to the tail base of the operated mice, 1 week and 2 weeks after surgery. Control mice received PBS injections instead.

In vivo imaging of lymphatic vessel function. To assess lymphatic vessel transport noninvasively, we used a Zeiss StereoLumar V12 stereomicroscope adapted for NIR imaging (17). A Harvard Apparatus PHD2000 syringe pump (Harvard Bioscience) and a custom-made intradermal catheter consisting of PE10 tubing and a 30-gauge needle were used for controlled dye administration. For evaluation of the transport function, a controlled infusion of $5 \mu 1$ of the NIR dye P20D800 (20 kDa PEGylated IRDye800) (17) was performed at a rate of $0.5 \mu \mathrm{l} / \mathrm{min}$ for 10 minutes at a site $1 \mathrm{~cm}$ proximal to the tail tip. Simultaneously, a video of the region near the surgical excision margin was recorded for a total of 20 minutes. To evaluate the functionality of the lymphatic vessels, fluorescence intensity was measured at $\mathrm{t}=20$ minutes in a selected region of interest $1.5-2 \mathrm{~cm}$ distal to the surgical excision margin using Zeiss AxioVision software.

Statistics. All graphs represent the mean \pm SD. Means of 2 groups were compared with the 2-tailed Student's $t$ test. Means of 3 or more groups were analyzed with 1-way or 2-way ANOVA with Tukey's post-hoc multiple comparison test or Bonferroni post-hoc test, respectively. Analyses were performed using GraphPad Prism V6.0 (GraphPad Software). Graphs are presented either as dot plots or as box and whisker plots, where the hinges represent the $25 \%-75 \%$ data range, the line represents the sample median, and the whiskers depict the minimum and maximum values. All experiments have been conducted at least in duplicate unless otherwise mentioned. $P<0.05$ was accepted as statistically significant.

Study approval. The study design including human samples was reviewed and accepted by the Ethical Committee of the Papageorgiou Hospital, Aristotle University of Thessaloniki, Greece. All patients provided written consent prior to inclusion in the study. All mice were housed under specific pathogen-free conditions, and the procedures were approved by Kantonales Veterinäramt Zürich (License number 225/2013). 


\section{Author contributions}

EG designed the experiments, acquired and analyzed data, and wrote the manuscript. STP conducted experiments and supported the design of experiments. SBB conducted experiments. JS conducted experiments, acquired and analyzed data. DD and ED provided reagents. $\mathrm{CH}$ designed experiments, LD conducted experiments and assisted with writing of the manuscript. MD designed experiments, supervised the study, and revised the manuscript.

\section{Acknowledgments}

This work was supported by the Swiss National Science Foundation grant 310030B_147087, European Research Council grant LYVICAM, Oncosuisse, Krebsliga Zurich (all to M.D.), and the ETH Zurich (to M.D. and E.G.). The authors would like to thank Babak Mehrara and Daniel Cuzzone (Memorial Sloan Kettering, New York, New York, USA) for technical advice regarding the lymphedema model; Tim Sparwasser (TWINCORE, Hannover, Germany) for providing the DEREG mice; Catharine Aquino and Lennart Opitz from the Functional Genomic Center Zurich (FGCZ) for the technical support with the RNA sequencing; Carlos Ochoa Pereira, the staff of the HCI Rodent Center, and Aretussa Apladas from the ETH Phenomic Center (EPIC) for excellent assistance with animal care; Kristian Ikenberg and Christiane Mittmann, Department of Pathology, University Hospital Zurich for helpful advice and technical assistance; and the ETH Scientific Center for Optical and Electron Microscopy (ScopeM) for assistance with microscopy.

Address correspondence to: Michael Detmar, Institute of Pharmaceutical Sciences, Swiss Federal Institute of Technology, ETH Zurich, Vladimir-Prelog-Weg 3, HCI H303, CH-8093 Zurich, Switzerland. Phone: 41.44.633.7361; E-mail: michael.detmar@pharma.ethz.ch.

1. Rockson SG. Secondary lymphedema: is it a primary disease? Lymphat Res Biol. 2008;6(2):63-64.

2. Warren AG, Brorson H, Borud LJ, Slavin SA. Lymphedema: a comprehensive review. Ann Plast Surg. 2007;59(4):464-472.

3. McLaughlin SA, et al. Prevalence of lymphedema in women with breast cancer 5 years after sentinel lymph node biopsy or axillary dissection: objective measurements. J Clin Oncol. 2008;26(32):5213-5219.

4. Stanton AW, Modi S, Mellor RH, Levick JR, Mortimer PS. Recent advances in breast cancer-related lymphedema of the arm: lymphatic pump failure and predisposing factors. Lymphat Res Biol. 2009;7(1):29-45.

5. Gousopoulos E, Proulx ST, Scholl J, Uecker M, Detmar M. Prominent lymphatic vessel hyperplasia with progressive dysfunction and distinct immune cell infiltration in lymphedema. Am J Pathol. 2016;186(8):2193-2203.

6. Ogata F, et al. Excess lymphangiogenesis cooperatively induced by macrophages and CD4(+) T cells drives the pathogenesis of lymphedema. J Invest Dermatol. 2016;136(3):706-714

7. Tabibiazar R, et al. Inflammatory manifestations of experimental lymphatic insufficiency. PLoS Med. 2006;3(7):e254.

8. Zampell JC, Yan A, Elhadad S, Avraham T, Weitman E, Mehrara BJ. CD4(+) cells regulate fibrosis and lymphangiogenesis in response to lymphatic fluid stasis. PLoS One. 2012;7(11):e49940.

9. Huggenberger R, et al. An important role of lymphatic vessel activation in limiting acute inflammation. Blood. 2011;117(17):4667-4678.

10. Baluk $\mathrm{P}$, et al. Pathogenesis of persistent lymphatic vessel hyperplasia in chronic airway inflammation. J Clin Invest. 2005;115(2):247-257.

11. Guo R, et al. Inhibition of lymphangiogenesis and lymphatic drainage via vascular endothelial growth factor receptor 3 blockade increases the severity of inflammation in a mouse model of chronic inflammatory arthritis. Arthritis Rheum. 2009;60(9):2666-2676.

12. Kataru RP, et al. T lymphocytes negatively regulate lymph node lymphatic vessel formation. Immunity. 2011;34(1):96-107.

13. Kim H, Kataru RP, Koh GY. Inflammation-associated lymphangiogenesis: a double-edged sword? J Clin Invest. 2014;124(3):936-942.

14. Shin K, et al. TH2 cells and their cytokines regulate formation and function of lymphatic vessels. Nat Commun. 2015;6:6196

15. Clavin NW, et al. TGF-beta1 is a negative regulator of lymphatic regeneration during wound repair. Am J Physiol Heart Circ Physiol. 2008;295(5):H2113-H2127.

16. Kataru RP, et al. Critical role of $\mathrm{CD} 11 \mathrm{~b}^{+}$macrophages and VEGF in inflammatory lymphangiogenesis, antigen clearance, and inflammation resolution. Blood. 2009;113(22):5650-5659.

17. Proulx ST, et al. Use of a PEG-conjugated bright near-infrared dye for functional imaging of rerouting of tumor lymphatic drainage after sentinel lymph node metastasis. Biomaterials. 2013;34(21):5128-5137.

18. Oka M, et al. Inhibition of endogenous TGF-beta signaling enhances lymphangiogenesis. Blood. 2008;111(9):4571-4579.

19. Boyman O, Kovar M, Rubinstein MP, Surh CD, Sprent J. Selective stimulation of T cell subsets with antibody-cytokine immune complexes. Science. 2006;311(5769):1924-1927.

20. Schirger A, Harrison EG, Janes JM. Idiopathic lymphedema. Review of 131 cases. JAMA. 1962;182:14-22.

21. Avraham T, et al. Th2 differentiation is necessary for soft tissue fibrosis and lymphatic dysfunction resulting from lymphedema. FASEB J. 2013;27(3):1114-1126.

22. Sather BD, et al. Altering the distribution of Foxp3(+) regulatory T cells results in tissue-specific inflammatory disease. $J$ Exp 
Med. 2007;204(6):1335-1347.

23. Himmel ME, Yao Y, Orban PC, Steiner TS, Levings MK. Regulatory T-cell therapy for inflammatory bowel disease: more questions than answers. Immunology. 2012;136(2):115-122.

24. Villalta SA, et al. Regulatory T cells suppress muscle inflammation and injury in muscular dystrophy. Sci Transl Med. 2014;6(258):258ra142.

25. Kim JM, Rasmussen JP, Rudensky AY. Regulatory T cells prevent catastrophic autoimmunity throughout the lifespan of mice. Nat Immunol. 2007;8(2):191-197.

26. Burzyn D, Benoist C, Mathis D. Regulatory T cells in nonlymphoid tissues. Nat Immunol. 2013;14(10):1007-1013.

27. Rosalia RA, Arenas-Ramirez N, Bouchaud G, Raeber ME, Boyman O. Use of enhanced interleukin-2 formulations for improved immunotherapy against cancer. Curr Opin Chem Biol. 2014;23:39-46.

28. Webster $\mathrm{KE}$, et al. In vivo expansion of T reg cells with IL-2-mAb complexes: induction of resistance to EAE and long-term acceptance of islet allografts without immunosuppression. $J$ Exp Med. 2009;206(4):751-760.

29. Koreth J, et al. Interleukin-2 and regulatory T cells in graft-versus-host disease. N Engl J Med. 2011;365(22):2055-2066.

30. Saadoun D, et al. Regulatory T-cell responses to low-dose interleukin-2 in HCV-induced vasculitis. $N$ Engl J Med. 2011;365(22):2067-2077.

31. Roncarolo MG, Battaglia M. Regulatory T-cell immunotherapy for tolerance to self antigens and alloantigens in humans. Nat Rev Immunol. 2007;7(8):585-598.

32. June CH, Blazar BR. Clinical application of expanded CD4+25+ cells. Semin Immunol. 2006;18(2):78-88.

33. Safinia N, Leech J, Hernandez-Fuentes M, Lechler R, Lombardi G. Promoting transplantation tolerance; adoptive regulatory T cell therapy. Clin Exp Immunol. 2013;172(2):158-168.

34. Singer BD, King LS, D'Alessio FR. Regulatory T cells as immunotherapy. Front Immunol. 2014;5:46.

35. Baluk $\mathrm{P}$, et al. TNF-alpha drives remodeling of blood vessels and lymphatics in sustained airway inflammation in mice. J Clin Invest. 2009;119(10):2954-2964.

36. Moore KW, de Waal Malefyt R, Coffman RL, O'Garra A. Interleukin-10 and the interleukin-10 receptor. Annu Rev Immunol. 2001;19:683-765

37. Panduro M, Benoist C, Mathis D. Tissue Tregs. Annu Rev Immunol. 2016;34:609-633.

38. Burzyn D, et al. A special population of regulatory T cells potentiates muscle repair. Cell. 2013;155(6):1282-1295.

39. Arpaia N, et al. A distinct function of regulatory T cells in tissue protection. Cell. 2015;162(5):1078-1089.

40. Nakamura K, Radhakrishnan K, Wong YM, Rockson SG. Anti-inflammatory pharmacotherapy with ketoprofen ameliorates experimental lymphatic vascular insufficiency in mice. PLoS ONE. 2009;4(12):e8380.

41. Campbell DJ. Control of regulatory T cell migration, function, and homeostasis. J Immunol. 2015;195(6):2507-2513.

42. Dudda JC, Perdue N, Bachtanian E, Campbell DJ. Foxp3 ${ }^{+}$regulatory T cells maintain immune homeostasis in the skin. $J$ Exp Med. 2008;205(7):1559-1565.

43. Siegmund K, et al. Migration matters: regulatory T-cell compartmentalization determines suppressive activity in vivo. Blood. 2005;106(9):3097-3104.

44. Bolger AM, Lohse M, Usadel B. Trimmomatic: a flexible trimmer for Illumina sequence data. Bioinformatics. 2014;30(15):2114-2120.

45. Li B, Dewey CN. RSEM: accurate transcript quantification from RNA-Seq data with or without a reference genome. BMC Bioinformatics. 2011;12:323.

46. Love MI, Huber W, Anders S. Moderated estimation of fold change and dispersion for RNA-seq data with DESeq2. Genome Biol. 2014;15(12):550. 